\title{
BILINEAR SPHERICAL MAXIMAL FUNCTIONS OF PRODUCT TYPE
}

\author{
LUZ RONCAL, SAURABH SHRIVASTAVA AND KALACHAND SHUIN
}

\begin{abstract}
In this paper we introduce and study a bilinear spherical maximal function of product type in the spirit of bilinear Calderón-Zygmund theory. This operator is different from the bilinear spherical maximal function considered by Geba et al. in [14]. We deal with lacunary and full versions of this operator, and prove weighted estimates with respect to genuine bilinear weights beyond the Banach range. Our results are implied by sharp sparse domination for both the operators, following ideas by Lacey [20]. In the case of the lacunary maximal operator we also use interpolation of analytic families of operators to address the weighted boundedness for the whole range of tuples.
\end{abstract}

\section{INTRODUCTION}

The theory of multilinear operators has been an active area of research for the past two decades in harmonic analysis. It finds its roots in the pioneer work by Coifman and Meyer [7], although it was the remarkable proof of the boundedness of the bilinear Hilbert transform by Lacey and Thiele $[22,23]$ that renewed the motivation for the study of multilinear singular integrals. The multilinear Calderón-Zygmund operators were systematically treated in [16] and later on, in [25], Lerner et al. developed an appropriate theory of multilinear maximal functions and multilinear weights. In particular, they established weighted boundedness for multilinear Calderón-Zygmund operators. Since then there have been several developments in the weighted theory of multilinear weights, we emphasize the recent works $[27,30]$ and references therein.

For notational convenience we shall restrict ourselves to the bilinear setting in this paper. Given locally integrable functions $f_{1}$ and $f_{2}$ defined on $\mathbb{R}^{n}$, the bilinear maximal function $\mathcal{M}\left(f_{1}, f_{2}\right)$ is defined by

$$
\mathcal{M}\left(f_{1}, f_{2}\right)(x):=\sup _{Q \ni x} \prod_{i=1}^{2} \frac{1}{|Q|} \int_{Q}\left|f_{i}\left(y_{i}\right)\right| d y_{i},
$$

where the supremum in the above is taken over all cubes $Q$ in $\mathbb{R}^{n}$ containing the point $x$. The cubes are always assumed to have their sides parallel to coordinate axes.

Note that the bilinear maximal operator $\mathcal{M}$ is dominated by the product of the classical Hardy-Littlewood maximal functions in a pointwise manner, i.e.,

$$
\mathcal{M}\left(f_{1}, f_{2}\right) \leq M\left(f_{1}\right) M\left(f_{2}\right),
$$

where $M$ denotes the Hardy-Littlewood maximal operator given by

$$
M(f)(x):=\sup _{Q \ni x} \frac{1}{|Q|} \int_{Q}|f(y)| d y .
$$

Let $1<p_{1}, p_{2}<\infty$ and $p$ be such that $\frac{1}{p}=\frac{1}{p_{1}}+\frac{1}{p_{2}}$. Hölder's inequality yields that the operator $\mathcal{M}$ is bounded from $L^{p_{1}}\left(w_{1}\right) \times L^{p_{2}}\left(w_{2}\right) \rightarrow L^{p}(w)$ for all $w_{i} \in A_{p_{i}}, i=1,2$, and $w=\prod_{j=1}^{2} w_{j}^{p / p_{j}}$. Here $A_{p}$ denotes the class of Muckenhoupt weights, see Subsection 3.1.

In [25], the authors showed that the bilinear maximal operator $\mathcal{M}$ is the appropriate analogue of the classical Hardy-Littlewood maximal operator. They introduced a suitable analogue of Muckenhoupt weights in the bilinear setting, the class $A_{\vec{P}}$ (see Subsection 3.1), and showed that the class $A_{\vec{P}}$ is bigger than the product of corresponding linear $A_{p}$ classes. The class $A_{\vec{P}}$ characterizes the weighted boundedness of the bilinear maximal operator $\mathcal{M}$. Moreover, the

Date: September 3, 2021.

2010 Mathematics Subject Classification. Primary 42B25, Secondary 46E35.

Key words and phrases. Bilinear spherical maximal functions, Bilinear weights, Sparse forms. 
bilinear Calderón-Zygmund operators possess weighted boundedness with respect to bilinear weights in $A_{\vec{P}}$. We refer the reader to [10, 24, 25] for more details.

Later on, first in [27] and then in [26,30], the notion of bilinear (or multilinear) weights was further generalised and extrapolation results were proved, see Subsection 3.1.

Motivated from the discussions above, in this paper we introduce a bilinear spherical maximal function of product type in the spirit of Calderón-Zygmund theory and investigate its weighted boundedness with respect to the bilinear weights just mentioned.

1.1. Linear spherical maximal functions and bilinear product-type analogues. Let $f: \mathbb{R}^{n} \rightarrow \mathbb{C}$ be a measurable function. Consider the average of $f$ over the sphere of radius $0<r<\infty$ given by

$$
\mathcal{A}_{r} f(x)=\int_{\mathbb{S}^{n-1}} f(x-r y) d \sigma_{n-1}(y),
$$

where $d \sigma_{n-1}$ is the normalized rotation invariant surface measure on the sphere $\mathbb{S}^{n-1}:=\{x \in$ $\left.\mathbb{R}^{n}:\|x\|=1\right\}$. The spherical maximal function was introduced by Stein [32] and is defined as

$$
M_{\text {full }}(f)(x):=\sup _{r>0} \mathcal{A}_{r} f(x), \quad x \in \mathbb{R}^{n} .
$$

Stein proved that $M_{\text {full }}$ is bounded in $L^{p}\left(\mathbb{R}^{n}\right)$ if and only if $\frac{n}{n-1}<p \leq \infty$ for all $n \geq 3$. The problem in dimension $n=2$ was settled later by Bourgain [4] (we refer to [29] for a different proof of Bourgain's result).

The dyadic or lacunary version of the spherical maximal function results by taking the supremum over the set $\left\{2^{j}: j \in \mathbb{Z}\right\}$, i.e.,

$$
M_{\text {lac }}(f)(x)=\sup _{j \in \mathbb{Z}} \mathcal{A}_{2^{j}} f(x) .
$$

The lacunary spherical maximal operator $M_{\text {lac }}$ is bounded in $L^{p}\left(\mathbb{R}^{n}\right)$ for all $1<p \leq \infty$ and $n \geq 2$, see $[6,8]$ for details. Weighted boundedness properties of the spherical maximal operators have been studied in $[9,12,13,28]$.

In a recent article, Lacey [20] revisited the spherical maximal function and, using a new approach that unified the lacunary and full versions, he managed to prove sparse bounds for these operators which led him to obtain new weighted norm inequalities. We also refer to [20] for a discussion about the suitability of $A_{p}$ weights in the context of the spherical maximal function.

In this paper we introduce a bilinear analogue of the spherical maximal function in the spirit of the bilinear Hardy-Littlewood maximal function (1), which plays a key role in the theory of bilinear Calderón-Zygmund operators. Define

$$
\mathcal{M}_{\text {full }}\left(f_{1}, f_{2}\right)(x):=\sup _{t>0} \mathcal{A}_{t} f_{1} \mathcal{A}_{t} f_{2}(x) \text {. }
$$

As earlier, if we take the supremum in the above over the dyadic numbers, we get bilinear analogue of the lacunary spherical maximal function. This way, the bilinear lacunary spherical maximal operator $\mathcal{M}_{\text {lac }}$ is defined as

$$
\mathcal{M}_{\text {lac }}\left(f_{1}, f_{2}\right)(x):=\sup _{j \in \mathbb{Z}} \mathcal{A}_{2^{j}} f_{1} \mathcal{A}_{2^{j}} f_{2}(x) .
$$

We refer to these operators as bilinear spherical maximal functions of product type ${ }^{1}$.

Note that $\mathcal{M}_{\text {full }}\left(f_{1}, f_{2}\right)$ (and $\mathcal{M}_{\text {lac }}\left(f_{1}, f_{2}\right)$ ) is dominated by the product of the linear full (respectively lacunary) spherical maximal functions in a pointwise sense. Therefore, Hölder's inequality immediately yields the $L^{p_{1}} \times L^{p_{2}} \rightarrow L^{p}$ estimates for the operators $\mathcal{M}_{\text {full }}$ and $\mathcal{M}_{\text {lac }}$. In fact, we also get the weighted estimates for the operator with respect to product weights, see Theorem 5.3. We will prove new weighted estimates for the bilinear spherical maximal functions with respect to bilinear weights that are beyond the type of weights as described in Theorem 5.3. This result is stated in Theorem 2.1. We exploit the ideas from [20] and establish a sparse domination principle for the bilinear spherical maximal functions in Theorem 2.3 so

\footnotetext{
${ }^{1}$ In a private communication with the second and third authors, Loukas Grafakos suggested that the terminology for this operator should be instead maximal product of spherical averages to better portray the nature of the operators.
} 

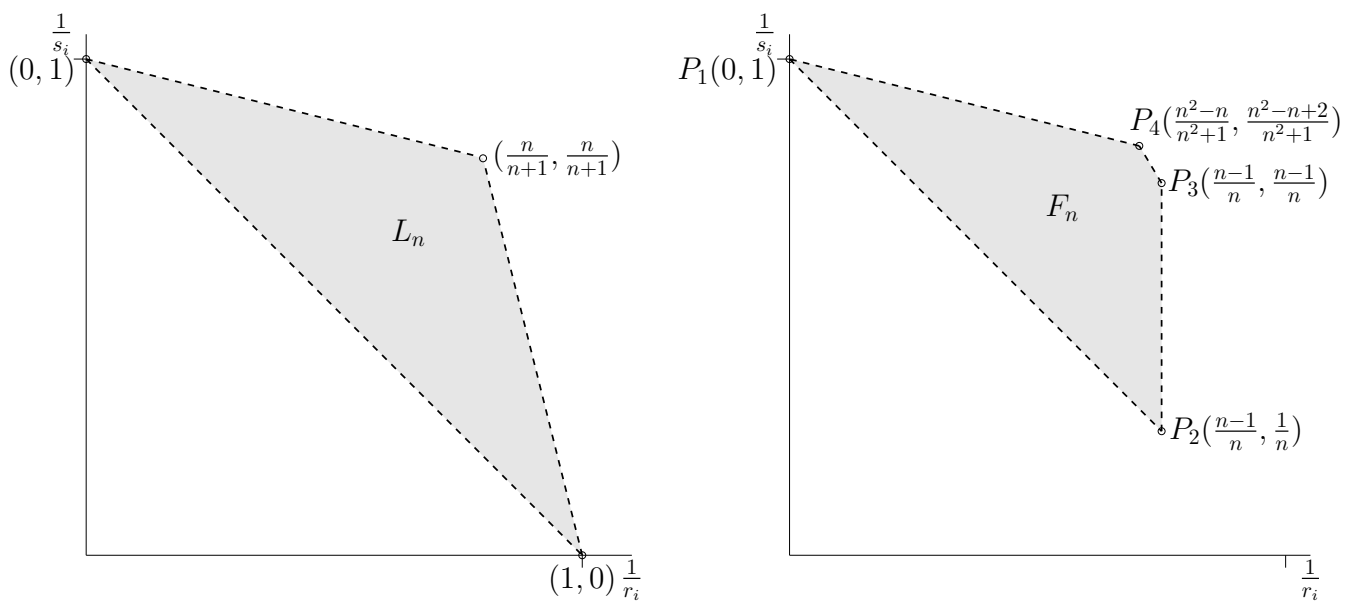

Figure 1. Triangle $L_{n}$ on the left and trapezium $F_{n}$ on the right.

that we deduce weighted estimates as a consequence of known results in the literature. These weighted estimates cover the best possible range of tuples $\left(p_{1}, p_{2}, p\right)$ for the $L^{p}$ boundedness of $\mathcal{M}_{\text {full }}$. Nevertheless, there is a dimensional constraint in this range of $\left(p_{1}, p_{2}, p\right)$ that prevents the operator $\mathcal{M}_{\text {lac }}$ from getting weighted boundedness in the range $1<p_{1}, p_{2}<\infty$ via Theorem 2.3. This restriction is overcome in Theorems 2.4 and 2.5. We also provide weighted boundedness of operators $\mathcal{M}_{\text {full }}$ and $\mathcal{M}_{\text {lac }}$ for the case of power weights in Corollaries 5.4, 5.5, and Theorem 2.4. This makes it easier to compare our results with the Hölder type results, see Section 5.

A different analogue of the spherical maximal function in the bilinear setting has been studied in the literature. It was introduced in [14] and is defined as follows:

$$
\mathcal{M}_{\mathrm{sph}}\left(f_{1}, f_{2}\right)(x):=\sup _{t>0} \int_{\mathbb{S}^{2 n-1}}\left|f_{1}(x-t y) f_{2}(x-t z)\right| d \sigma_{2 n-1}(y, z) .
$$

In $[3,15,17]$ the authors proved partial results obtaining $L^{p_{1}} \times L^{p_{2}} \rightarrow L^{p}$ estimates for the operator $\mathcal{M}_{\mathrm{sph}}$ for a certain range of $p_{1}, p_{2}$ and $p$ and some assumptions on the dimension $n$. In [18] the authors proved the following pointwise domination result

$$
\mathcal{M}_{\text {sph }}\left(f_{1}, f_{2}\right)(x) \lesssim M_{\text {full }}\left(f_{1}\right)(x) M\left(f_{2}\right)(x),
$$

and extended the $L^{p_{1}} \times L^{p_{2}} \rightarrow L^{p}$ estimates for the operator $\mathcal{M}_{\mathrm{sph}}$ to the best possible range of exponents $p_{1}, p_{2}$ and $p$ for all $n \geq 2$ (note that an estimate similar to (3) holds with the roles of $M_{\text {full }}$ and $M$ interchanged due to symmetry). We also refer to the recent papers $[1,11]$ for the generalisation of the bilinear spherical maximal function to the multilinear setting. Weighted estimates for the bilinear maximal operator $\mathcal{M}_{\text {sph }}$ defined in (2) beyond the ones that can be obtained trivially from the pointwise estimate (3) remain as an open problem.

The paper is organised as follows. We state the main results in the next section, then in Section 3 we recall necessary definitions and results and also set notation that we use in the paper. Section 4 is devoted to prove weighted estimates for the operators under consideration and we complete the proofs of Theorems 2.1, 2.4, and 2.5 in this section. In Section 5 we discuss some examples comparing the weighted results obtained in Theorem 2.1 with the Hölder type results. Next, in Section 6 we give the proof of sparse domination result Theorem 2.3. Finally, in Section 7 we provide the necessity of some conditions for such a sparse domination.

\section{MAIN RESUlts}

Our first main result is the following theorem containing weighted estimates for the product type operators with bilinear weights in the class $A_{\vec{q}, \vec{r}}$ (see Definition 3.2). In what follows, we will denote by $L_{n}$ the triangle with vertexes $(0,1),(1,0)$ and $\left(\frac{n}{n+1}, \frac{n}{n+1}\right)$ and by $F_{n}$ the trapezium with vertexes $(0,1),\left(\frac{n-1}{n}, \frac{1}{n}\right),\left(\frac{n-1}{n}, \frac{n-1}{n}\right)$ and $\left(\frac{n^{2}-n}{n^{2}+1}, \frac{n^{2}-n+2}{n^{2}+1}\right)$, see Figure 1 . 
Theorem 2.1. Let $n \geq 2$. For $i=1,2$, let $\left(\frac{1}{r_{i}}, \frac{1}{s_{i}}\right)$ be in the interior of $L_{n}$ (respectively $\left.F_{n}\right)$. Assume that $t:=\frac{s_{1} s_{2}}{s_{1}+s_{2}-s_{1} s_{2}}>1$. Then for all $\vec{q}=\left(q_{1}, q_{2}\right), \frac{1}{q}=\frac{1}{q_{1}}+\frac{1}{q_{2}}$ with $r_{i} \leq q_{i}$, $i=1,2$, and $t^{\prime}>q$, the operator $\mathcal{M}_{\text {lac }}$ (respectively $\mathcal{M}_{\text {full }}$ ) extends to a bounded operator from $L^{q_{1}}\left(w_{1}\right) \times L^{q_{2}}\left(w_{2}\right) \rightarrow L^{q}(w)$, i.e.,

$$
\left\|\mathcal{M}\left(f_{1}, f_{2}\right)\right\|_{L^{q}(w)} \leq C\left([\vec{w}]_{A_{\vec{q}, \vec{r}}}\right) \prod_{i=1}^{2}\left\|f_{i}\right\|_{L^{q_{i}}\left(w_{i}\right)},
$$

where $\mathcal{M}:=\mathcal{M}_{\text {lac }}$ (respectively $\left.\mathcal{M}_{\text {full }}\right), \vec{w}=\left(w_{1}, w_{2}\right) \in A_{\vec{q}, \vec{r}}$ with $\vec{r}=\left(r_{1}, r_{2}, t\right)$ defined as in Definition 3.2 and $C\left([\vec{w}]_{A_{\vec{q}, \vec{r}}}\right)$ is a constant depending on the characteristic of the weight.

Remark 2.2. Observe that, in Theorem 2.1, we can consider $\vec{q}=\left(q_{1}, q_{2}\right)$ with $q<1$ as well, which means that the weighted inequalities hold beyond the Banach range.

The weighted estimates in Theorem 2.1 are indeed consequence of a sparse domination principle for the bilinear spherical maximal functions shown in Theorem 2.3 below and the extrapolation result in [27, Theorem 1.1]. Actually, one could state an improved result, providing the quantitative bounds (i.e., giving more explicit information on $C\left([\vec{w}]_{A_{\vec{q}, \vec{r}}}\right)$ ), including end-points, and vector-valued inequalities, see Theorem 4.3 and Remark 4.4. For these consequences we appeal to $[26,27,30]$.

Before stating the sparse domination result let us set up the notation. A collection of cubes $\mathcal{S}$ in $\mathbb{R}^{n}$ is said to be $\eta$-sparse, $0<\eta<1$, if there are sets $\left\{E_{S} \subset S: S \in \mathcal{S}\right\}$ which are pairwise disjoint and satisfy $\left|E_{S}\right|>\eta|S|$ for all $S \in \mathcal{S}$. By the term $(p, q, r)$-sparse form we mean:

$$
\Lambda_{\mathcal{S}_{p, q, r}}(f, g, h):=\sum_{S \in \mathcal{S}}|S|\langle f\rangle_{S, p}\langle g\rangle_{S, q}\langle h\rangle_{S, r}
$$

see Section 3 for notations.

Theorem 2.3. Let $n \geq 2$. For $i=1,2$, let $\left(\frac{1}{r_{i}}, \frac{1}{s_{i}}\right)$ be in the interior of $L_{n}$ (respectively $F_{n}$ ) and $\rho_{i}>r_{i}$. Then for any non-negative compactly supported bounded functions $f_{1}, f_{2}$ and $h$, there exists a sparse collection $\mathcal{S}=\mathcal{S}_{\rho_{1}, \rho_{2}, t}$ such that

$$
\left\langle\mathcal{M}\left(f_{1}, f_{2}\right), h\right\rangle \leq C \Lambda_{\mathcal{S}_{\rho_{1}, \rho_{2}, t}}\left(f_{1}, f_{2}, h\right)
$$

where $t:=\frac{s_{1} s_{2}}{s_{1}+s_{2}-s_{1} s_{2}}>1$ and $\mathcal{M}:=\mathcal{M}_{\text {lac }}$ (respectively $\left.\mathcal{M}_{\text {full }}\right)$.

Furthermore, the ranges of $\left(\frac{1}{r_{i}}, \frac{1}{s_{i}}\right), i=1,2$, are sharp up to endpoints in the sense that no such result can hold if both $\left(\frac{1}{r_{i}}, \frac{1}{s_{i}}\right), i=1,2$, do not lie in the closure of $L_{n}$ (respectively $F_{n}$ ). The condition $1<t<\infty$ is also a necessary condition.

We prove the sufficiency part of this theorem in two steps. First, we shall establish an analogous result for characteristic functions. Then we consider the theorem for general functions. In this second stage, we use a recursive argument in which both functions involved in the bilinear sparse form are decomposed into simple functions. We perform this step by taking one of the functions to be a characteristic function, then keeping this function fixed we decompose the other function into suitable simple functions. This process along with an application of Carleson embedding theorem allows us to obtain sparse domination for general compactly supported bounded function in one place whereas the other function is taken to be a characteristic function. We repeat the procedure with the second function. The proof of these results and of Theorem 2.3 will be given in Section 6 and in Section 7 (in the latter we prove the necessity of the conditions for the sparse domination.

Note that in Theorem 2.3 we have the necessary condition $1<t:=\frac{s_{1} s_{2}}{s_{1}+s_{2}-s_{1} s_{2}}<\infty$. This condition translates into the fact that we cannot choose both $r_{1}, r_{2} \leq \frac{2 n}{2 n-1}$. Thus, in the case of lacunary spherical maximal operator $\mathcal{M}_{\text {lac }}$ in Theorem 2.1, we cannot consider both $q_{1}, q_{2} \leq \frac{2 n}{2 n-1}$ simultaneously. Nevertheless, we cover the complete range of exponents for the operator $\mathcal{M}_{\text {lac }}$ using a different method. We establish non-trivial weighted estimates for $\mathcal{M}_{\text {lac }}$ for tuples $\left(q_{1}, q_{2}, q\right)$ with $1<q_{1}, q_{2} \leq \frac{2 n}{2 n-1}$ and $\frac{1}{q_{1}}+\frac{1}{q_{2}}=\frac{1}{q}$, see Theorem 2.4 and Theorem 2.5. We exploit ideas from $[19,31]$ to obtain these results, based on interpolation of analytic families of linear operators in [5]. 
Observe that this scenario does not occur in the case of $\mathcal{M}_{\text {full }}$. Indeed, the condition $r_{1}, r_{2}>$ $\frac{n}{n-1}$ holds necessarily for the operator $\mathcal{M}_{\text {full }}$. Therefore, Theorem 2.3 addresses the question of weighted boundedness of the operator $\mathcal{M}_{\text {full }}$ with respect to genuine weights $\vec{w}=\left(w_{1}, w_{2}\right) \in A_{\vec{q}, \vec{r}}$ for the entire possible range of tuples $\left(q_{1}, q_{2}, q\right)$.

As mentioned previously, we shall establish non-trivial weighted estimates for $\mathcal{M}_{\text {lac }}$ for the tuple $\left(p_{1}, p_{2}, p\right)$ with $1<p_{1}, p_{2} \leq \frac{2 n}{2 n-1}$ and $\frac{1}{p_{1}}+\frac{1}{p_{2}}=\frac{1}{p}$. For the sake of simplicity we consider $p_{1}=p_{2}=p$ in the following theorem, since the statement of the result for general tuples turns out to be rather cumbersome.

Theorem 2.4. Let $n \geq 2$. The operator $\mathcal{M}_{\mathrm{lac}}$ is bounded from $L^{p}\left(|x|^{\alpha}\right) \times L^{p}\left(|x|^{\beta}\right)$ to $L^{p / 2}\left(|x|^{\frac{\alpha+\beta}{2}}\right)$ with $1<p \leq \frac{2 n}{2 n-1}$ for $\alpha, \beta$ satisfying:

$$
(1-n) p<\alpha, \beta<(n-1)(p-1) \quad \text { and } \quad \alpha+\beta>2(1-n)(n-(n-1) p), \quad n \geq 2 .
$$

We would like to remark that while proving Theorem 2.4, we actually get weighted boundedness of operators $\mathcal{M}_{\text {lac }}$ for the triplet $\left(p_{1}, p_{2}, p\right)$ for more general weights than considered in Theorem 2.4 above (see Theorem 2.5). Moreover, these weights do not come from the product type bilinear weights. This point is discussed in detail in Section 5 .

Let $\frac{1}{\phi_{\operatorname{lac}}\left(\frac{1}{r}\right)}$ denote the piecewise linear function on the interval $(0,1)$ whose graph connects the points $(0,1),\left(\frac{n}{n+1}, \frac{n}{n+1}\right)$ and $(1,0)$, i.e.,

$$
\frac{1}{\phi_{\text {lac }}\left(\frac{1}{r}\right)}= \begin{cases}1-\frac{1}{r n}, & \text { if } \quad 0<\frac{1}{r} \leq \frac{n}{n+1} \\ n\left(1-\frac{1}{r}\right), & \text { if } \quad \frac{n}{n+1}<\frac{1}{r}<1 .\end{cases}
$$

An inspection of the proof of Theorem 2.4 delivers the following (indeed, this is a byproduct of Step I in the proof), see Section 3 for the definitions of weights.

Theorem 2.5. Let $n \geq 2$. The operator $\mathcal{M}_{\mathrm{lac}}$ is bounded from $L^{p_{1}}\left(w_{1}\right) \times L^{p_{2}}\left(w_{2}\right)$ to $L^{p}(w)$, where $1<p_{1}, p_{2} \leq \frac{2 n}{2 n-1}$ and for certain weights $\vec{w}=\left(w_{1}, w_{2}\right)$ which do not belong to product type weights

$$
\bigcup_{1<r_{i}<p_{i}}(\prod_{i=1}^{2} A_{\frac{p_{i}}{r_{i}}} \cap \mathrm{RH} \underbrace{}_{\left(\frac{\phi_{1 \mathrm{cc}}^{\prime}\left(\frac{1}{r_{i}}\right)}{\tilde{p}_{i}}\right)^{\prime}}) \bigcup\left(\mathcal{R}_{p_{1}} \times \mathcal{R}_{p_{2}}\right),
$$

where

$$
\mathcal{R}_{p}=\left\{|x|^{b}: 1-n \leq b<(n-1)(p-1)\right\}, \quad n \geq 2 .
$$

\section{Notations And Definitions}

In this section we collect some of the notations and definitions that we use in this paper. With the letters $c, C \ldots$ we denote structural constants that depend only on the dimension and on parameters. Their values might vary from one occurrence to another, and in most of the cases we will not track the explicit dependence. We will write $\gamma_{1} \lesssim \gamma_{2}$ if $\gamma_{1} \leq c \gamma_{2}$ for a structural constant $c$. Given $p \geq 1$, the conjugate exponent of $p$ will be denoted by $p^{\prime}$, i.e., $1 / p+1 / p^{\prime}=1$.

For any cube $Q$ and $1<p<\infty$, we define

$$
\langle f\rangle_{Q, p}:=\left(\frac{1}{|Q|} \int_{Q}|f(x)|^{p} d x\right)^{1 / p}, \quad\langle f\rangle_{Q}:=\frac{1}{|Q|} \int_{Q}|f(x)| d x
$$

where $|Q|$ denotes the Lebesgue measure of $Q$.

A weight is a non-negative locally Lebesgue integrable function that is non-zero in a set of positive measure. We say that a weight $w$ belongs to the Muckenhoupt class $A_{p}$ if

$$
[w]_{A_{p}}:=\sup _{Q}\left(\frac{1}{|Q|} \int_{Q} w d x\right)\left(\frac{1}{|Q|} \int_{Q} w^{1-p^{\prime}} d x\right)^{p-1}<\infty, \quad 1<p<\infty .
$$

The quantity $[w]_{A_{p}}$ is referred to as the $A_{p}$ characteristic of $w \in A_{p}$. For $p=1$ the class $A_{1}$ consists of all $w$ such that

$$
[w]_{A_{1}}:=\operatorname{ess} \sup \frac{M(w)}{w}<\infty .
$$


Given $s>1$, a weight belongs to the reverse Hölder $\mathrm{RH}_{s}$ if there exists a constant $C$ such that, for every cube $Q$ in $\mathbb{R}^{n}$ with sides parallel to the coordinate axes,

$$
\left(\frac{1}{|Q|} \int_{Q} w^{s} d x\right)^{1 / s} \leq \frac{C}{|Q|} \int_{Q} w d x<\infty
$$

3.1. Bilinear weights. Let $1 \leqslant p_{1}, p_{2}<\infty$ and $p$ be such that

$$
\frac{1}{p}=\frac{1}{p_{1}}+\frac{1}{p_{2}} \text {. }
$$

Definition 3.1. [25, Definition 3.5] Let $\vec{p}=\left(p_{1}, p_{2}\right)$. For a given pair of weights $\vec{w}=\left(w_{1}, w_{2}\right)$, set $w:=\prod_{i=1}^{2} w_{i}^{p / p_{i}}$. We say that $\vec{w} \in A_{\vec{P}}$ if

$$
[\vec{w}]_{A_{\vec{P}}}:=\sup _{Q}\left(\frac{1}{|Q|} \int_{Q} w d x\right) \prod_{j=1}^{2}\left(\frac{1}{|Q|} \int_{Q} w_{j}^{1-p_{j}^{\prime}} d x\right)^{p / p_{j}^{\prime}}<\infty .
$$

When $p_{j}=1,\left(\frac{1}{|Q|} \int_{Q} w_{j}^{1-p_{j}^{\prime}}\right)^{1 / p_{j}^{\prime}}$ is understood as $\left(\inf _{Q} w_{j}\right)^{-1}$. The quantity $[\vec{w}]_{A_{\vec{P}}}$ is referred to as the bilinear $A_{\vec{P}}$ characteristic of the bilinear weight $\vec{w}$.

The bilinear $A_{\vec{P}}$ class was further generalised recently in [27].

Definition 3.2. [27, Section 1] Let $\vec{p}=\left(p_{1}, p_{2}\right)$ and $p$ be as in (6). For a tuple $\vec{r}=\left(r_{1}, r_{2}, r_{3}\right)$ with $r_{i} \leq p_{i}, i=1,2$, and $r_{3}^{\prime}>p$, where $1 \leq r_{1}, r_{2}, r_{3}<\infty$, we say that $\vec{w}=\left(w_{1}, w_{2}\right) \in A_{\vec{p}, \vec{r}}$ if $0<w_{i}<\infty$ a.e. for $i=1,2$ and

$$
[\vec{w}]_{A_{\vec{p}, \vec{r}}}:=\sup _{Q \subset \mathbb{R}^{n}}\left\langle w^{\frac{r_{3}^{\prime}}{r_{3}^{\prime}-p}}\right\rangle_{Q}^{\frac{1}{p}-\frac{1}{r_{3}^{\prime}}} \prod_{i=1}^{2}\left\langle w_{i}^{\frac{r_{i}}{r_{i}-p_{i}}}\right\rangle_{Q}^{\frac{1}{r_{i}}-\frac{1}{p_{i}}}<\infty,
$$

where $w:=\prod_{i=1}^{2} w_{i}^{p / p_{i}}$. When $r_{3}=1$, the term corresponding to $w$ needs to be replaced by $\langle w\rangle_{Q}^{1 / p}$. Analogously, when $p_{i}=r_{i}$, the term corresponding to $w_{i}$ needs to be replaced by $\operatorname{ess}_{\sup _{Q}} w_{i}^{-1 / p_{i}}$.

Remark 3.3. Note that $A_{\vec{p},(1,1,1)}$ agrees with the class $A_{\vec{P}}$.

The following result describes the bilinear weights $A_{\vec{p}, \vec{r}}$ in terms of the classical $A_{p}$ weights. This provides a useful tool in the study of weighted estimates with respect to bilinear weights.

Lemma 3.4. [27, Lemma 5.3] Let $\vec{p}=\left(p_{1}, p_{2}\right)$ with $1<p_{1}, p_{2}<\infty$ and $\vec{r}=\left(r_{1}, r_{2}, r_{3}\right)$ with $1 \leq r_{1}, r_{2}, r_{3}<\infty$. Let $p^{\prime}:=p_{3}$ and $\frac{1}{r}:=\sum_{i=1}^{3} \frac{1}{r_{i}}$. Assume that $r_{i} \leq p_{i}$ for $i=1,2$ and $r_{3}^{\prime}>p$. Consider

$$
\frac{1}{\delta_{i}}=\frac{1}{r_{i}}-\frac{1}{p_{i}} \quad \text { and } \quad \frac{1}{\theta_{i}}=\frac{1-r}{r}-\frac{1}{\delta_{i}}, \quad i=1,2,3 .
$$

Then $\vec{w}=\left(w_{1}, w_{2}\right) \in A_{\vec{p}, \vec{r}}$ if and only if

and

$$
w_{i}^{\frac{\theta_{i}}{p_{i}}} \in A_{\frac{1-r}{r} \theta_{i}} \quad \text { with } \quad\left[w_{i}^{\frac{\theta_{i}}{p_{i}}}\right]_{\frac{1-r}{r} \theta_{i}} \leq[\vec{w}]_{A_{\vec{p}, \vec{r}}}^{\theta_{i}}, \quad i=1,2
$$

$$
w^{\frac{\delta_{3}}{p}} \in A_{\frac{1-r}{r} \delta_{3}} \quad \text { with } \quad\left[w^{\frac{\delta_{3}}{p}}\right]_{\frac{1-r}{r} \delta_{3}} \leq[\vec{w}]_{A_{\vec{p}, \vec{r}}}^{\delta_{3}}
$$

In [30], Nieraeth presented an alternative approach to describe the bilinear weights $A_{\vec{p}, \vec{r}}$ and defined yet another class of weights that is equivalent to the class defined in [27]. Nieraeth extended the extrapolation results contained in [27] in several directions.

Definition 3.5. [30, Definition 2.1] Let $\vec{p}=\left(p_{1}, p_{2}\right), \vec{q}=\left(q_{1}, q_{2}\right)$ with $p_{1}, p_{2} \in(0, \infty)$ and $q_{1}, q_{2} \in(0, \infty]$. Let $q$ be given by $\frac{1}{q}=\frac{1}{q_{1}}+\frac{1}{q_{2}}$. We say $(\vec{p}, s) \leq \vec{q}$ if $\vec{p} \leq \vec{q}$ and $q \leq s$ where $s \in(0, \infty]$. Here $\vec{p} \leq \vec{q}$ means that $p_{i} \leq q_{i}, i=1,2$. For weights $w_{1}, w_{2}$ write $w=\prod_{i=1}^{2} w_{i}$. We say that $\vec{w}=\left(w_{1}, w_{2}\right) \in A_{\vec{q},(\vec{p}, s)}$ if

$$
[\vec{w}]_{\vec{q},(\vec{p}, s)}:=\sup _{Q}\left(\prod_{i=1}^{2}\left\langle w_{i}^{-1}\right\rangle_{\frac{1}{p_{i}}-\frac{1}{q_{i}}}, Q\langle w\rangle_{\frac{1}{q}-\frac{1}{s}}, Q\right)<\infty,
$$

where the supremum is taken over all cubes (with sides parallel to coordinate axes) in $\mathbb{R}^{n}$. 
Remark 3.6. Note that the definition above includes the case $q_{j}=\infty$. In this case the norm is interpreted as $\left\|f_{j}\right\|_{L^{q_{j}}\left(w_{j}^{q_{j}}\right)}=\left\|f_{j} w_{j}\right\|_{L^{\infty}}$. Also, the definition is used with $\frac{1}{q_{j}}=0$ when $q_{j}=\infty$. We refer to [30] for more details on this. We also would like to refer the reader to [26], where authors consider a slightly different approach to include the end-points cases which allows one or more indices to take value infinity. Further, note that when $q_{j}$ are finite, the following relation holds: $\left(w_{1}^{q_{1}}, w_{2}^{q_{2}}\right) \in A_{\vec{q},\left(r_{1}, r_{2}, t\right)}$ if and only if $\vec{w} \in A_{\vec{q},\left(\vec{r}, t^{\prime}\right)}$.

\section{Proofs of Weighted estimates}

4.1. Proof of Theorem 2.1. As pointed out earlier, the proof of Theorem 2.1 follows from the sparse domination result Theorem 2.3 and the already well-known consequences in the literature.

Theorem 4.1. [27, Corollary 2.15] Fix $\vec{r}=\left(r_{1}, r_{2}, r_{3}\right)$, with $r_{i} \geq 1$ and $\sum_{i=1}^{3} \frac{1}{r_{i}}>1$, and a sparsity constant $\eta \in(0,1)$. Let $T$ be an operator so that for every $f_{1}, f_{2}, h \in C_{c}^{\infty}\left(\mathbb{R}^{n}\right)$

$$
\left|\int_{\mathbb{R}^{n}} T\left(f_{1}, f_{2}\right)(x) h(x) d x\right| \lesssim \sup _{\mathcal{S}} \Lambda_{\mathcal{S}, \vec{r}}\left(f_{1}, f_{2}, h\right),
$$

where the supremum runs over all sparse families with sparsity constant $\eta$. Then for all exponents $\vec{q}=\left(q_{1}, q_{2}\right)$, with $r_{i}<q_{i}$ for $i=1,2$ and $r_{3}^{\prime}>q$ and all the weights $\vec{v}=\left(v_{1}, v_{2}\right) \in A_{\vec{q}, \vec{r}}$, and for all $f_{1}, f_{2}, h \in C_{c}^{\infty}\left(\mathbb{R}^{n}\right)$, we have

$$
\left\|T\left(f_{1}, f_{2}\right)\right\|_{L^{q}(v)} \lesssim \prod_{i=1}^{2}\left\|f_{i}\right\|_{L^{q_{i}\left(v_{i}\right)}},
$$

where $\frac{1}{q}=\frac{1}{q_{1}}+\frac{1}{q_{2}}$ and $v=v_{1}^{\frac{q}{q_{1}}} v_{2}^{\frac{q}{q_{2}}}$.

The sparse domination result contained in Theorem 2.3 yields the weighted estimates in Theorem 2.1 by using Theorem 4.1 and the extrapolation result in [27, Theorem 1.1].

4.2. Quantitative bounds in Theorem 2.1. In [30], an improvement of the quantitative bounds obtained from sparse domination in multilinear forms was achieved. Indeed, the results in [27] missed the quantitative weighted bounds for the range $q<1$.

Theorem 4.2. [30, Corollary 4.2] Let $T$ be a bilinear or positive valued bi-sublinear operator and assume that for some $p_{1}, p_{2} \in(0, \infty)$ and $t \in[1, \infty]$, we have the sparse domination of the bilinear operator for every $f_{1}, f_{2}, h \in C_{c}^{\infty}\left(\mathbb{R}^{n}\right)$, i.e.,

$$
\left|\int_{\mathbb{R}^{n}} T\left(f_{1}, f_{2}\right)(x) h(x) d x\right| \lesssim \sup _{\mathcal{S}} \Lambda_{\mathcal{S},\left(p_{1}, p_{2}, t\right)}\left(f_{1}, f_{2}, h\right),
$$

then for all $\vec{q}=\left(q_{1}, q_{2}\right)$ with $q_{1}, q_{2} \in(0, \infty]$ such that $\left(\vec{p}, t^{\prime}\right)<\vec{q}$ and all weights $\vec{w} \in A_{\vec{q},\left(\vec{p}, t^{\prime}\right)}$, the operator $T$ extends to a bounded operator $L^{q_{1}}\left(w_{1}^{q_{1}}\right) \times L^{q_{2}}\left(w_{2}^{q_{2}}\right) \rightarrow L^{q}\left(w^{q}\right)$, where $\frac{1}{q}=\frac{1}{q_{1}}+\frac{1}{q_{2}}$, with the bound

$$
\|T\|_{L^{q_{1}}\left(w_{1}^{q_{1}}\right) \times L^{q_{2}}\left(w_{2}^{q_{2}}\right) \rightarrow L^{q}\left(w^{q}\right)} \lesssim[\vec{w}]_{A_{\vec{q},\left(\vec{p}, t^{\prime}\right)}} \max \left(\frac{\frac{1}{p_{1}}}{\frac{1}{p_{1}}-\frac{1}{q_{1}}, \frac{\frac{1}{p_{2}}}{p_{2}}-\frac{1}{q_{2}}}, \frac{1-\frac{1}{t^{\prime}}}{\frac{1}{q}-\frac{1}{t^{\prime}}}\right) .
$$

In view of the theorem above, the sparse domination results obtained in Theorem 2.3 together with the extrapolation in [27, Theorem 1.1] yield the following improved weighted estimates for the operators $\mathcal{M}_{\text {lac }}$ and $\mathcal{M}_{\text {full }}$.

Theorem 4.3. Let $n \geq 2$ and $\left(\frac{1}{r_{i}}, \frac{1}{s_{i}}\right), i=1,2$, be in the interior of $L_{n}$ (respectively $F_{n}$ ). Assume that $t=\frac{s_{1} s_{2}}{s_{1}+s_{2}-s_{1} s_{2}}>1$. Then for all $\vec{q}=\left(q_{1}, q_{2}\right)$ with $q_{1}, q_{2} \in(0, \infty]$ such that $\vec{q}>\left(\vec{r}, t^{\prime}\right)$ the operator $\mathcal{M}_{\text {lac }}\left(\right.$ respectively $\left.\mathcal{M}_{\text {full }}\right)$ extends to a bounded operator $L^{q_{1}}\left(w_{1}^{q_{1}}\right) \times L^{q_{2}}\left(w_{2}^{q_{2}}\right) \rightarrow L^{q}\left(w^{q}\right)$, where $\frac{1}{q}=\frac{1}{q_{1}}+\frac{1}{q_{2}}$, with the bound

$$
\|\mathcal{M}\|_{L^{q_{1}}\left(w_{1}^{q_{1}}\right) \times L^{q_{2}}\left(w_{2}^{q_{2}}\right) \rightarrow L^{q}\left(w^{q}\right)} \lesssim[\vec{w}]_{A_{\vec{q},\left(\vec{r}, t^{\prime}\right)}}\left(\frac{\frac{1}{r_{1}}}{\frac{1}{r_{1}}-\frac{1}{q_{1}}}, \frac{\frac{1}{r_{2}}}{\frac{1}{r_{2}}-\frac{1}{q_{2}}}, \frac{1-\frac{1}{t^{\prime}}}{\frac{1}{q}-\frac{1}{t^{\prime}}}\right),
$$

where $\mathcal{M}:=\mathcal{M}_{\text {lac }}\left(\right.$ respectively $\left.\mathcal{M}_{\text {full }}\right)$ and $A_{\vec{q},\left(\vec{r}, t^{\prime}\right)}$, with $\left(\vec{r}, t^{\prime}\right)=\left(r_{1}, r_{2}, t^{\prime}\right)$, is defined as in Definition 3.5 . 
Remark 4.4. Note that the end-point extrapolation in $[26,30]$ allows the index $q_{j}$ above to take value infinity. Moreover, the original Theorem 4.1 contained in [27] includes vector-valued results. These apply to our sparse domination in Theorem 2.1, so that vector-valued inequalities are immediately obtained from [27, Corollary 2.15], see also [30, Corollary 4.6].

\subsection{Weighted boundedness for the triplet $\left(p_{1}, p_{2}, p\right)$.}

Proof of Theorem 2.4. The proof of Theorem 2.4 is done in two steps. The first step is to establish a more general result by using analytic interpolation for a family of bilinear operators. Then in the second step we use this general result with a suitable choice of exponents to deduce the theorem.

Step I: Let $1<\tilde{p}_{1}=\tilde{p}_{2} \leq \frac{2 n}{2 n-1}$. Now consider $\frac{2 n}{2 n-1}<p_{1}, p_{2}<\infty, \frac{1}{p}=\frac{1}{p_{1}}+\frac{1}{p_{2}}$, and $\left(\frac{1}{r_{i}}, \frac{1}{s_{i}}\right) \in L_{n}, i=1,2$ with $t=\frac{s_{1} s_{2}}{s_{1}+s_{2}-s_{1} s_{2}}>1$. For $\vec{r}=\left(r_{1}, r_{2}, t\right)<\vec{p}:=\left(p_{1}, p_{2}, p\right)$, let $\vec{w}=\left(w_{1}, w_{2}\right) \in A_{\vec{p}, \vec{r}}$. By Theorem 2.1 we have

$$
\left\|\mathcal{M}_{\text {lac }}\left(f_{1}, f_{2}\right)\right\|_{L^{p}(w)} \leq C_{1}\left\|f_{1}\right\|_{L^{p_{1}\left(w_{1}\right)}}\left\|f_{2}\right\|_{L^{p_{2}\left(w_{2}\right)}} .
$$

Also, note that by Theorem 5.3 we have the following weighted estimates for the product weights.

$$
\left\|\mathcal{M}_{\text {lac }}\left(f_{1}, f_{2}\right)\right\|_{L^{q}(v)} \leq C_{2}\left\|f_{1}\right\|_{L^{q_{1}}\left(v_{1}\right)}\left\|f_{2}\right\|_{L^{q_{2}\left(v_{2}\right)}},
$$

for $1<q_{i}<\tilde{p}_{i}, \frac{1}{q}=\frac{1}{q_{1}}+\frac{1}{q_{2}}, v_{i} \in\left(A_{\frac{q_{i}}{t_{i}}} \cap \mathrm{RH}_{\left(\frac{\phi_{\text {lac }}^{\prime}\left(\frac{1}{t_{i}}\right)}{q_{i}}\right)^{\prime}}\right) \cup \mathcal{R}_{q_{i}}, v=v_{1}^{\frac{q}{q_{1}}} v_{2}^{\frac{q}{q_{2}}}$ and $\left(\frac{1}{t_{i}}, \frac{1}{\eta_{i}}\right) \in L_{n}$ for some $\eta_{i} \in(1, \infty)$ and $1<t_{i}<q_{i}<\eta_{i}^{\prime}$, for $i=1,2$.

We consider the linearised operator $\mathcal{M}_{\text {lac }}$ as follows

$$
\mathcal{M}_{\text {lac }}\left(f_{1}, f_{2}\right)(x)=\mathcal{A}_{\tau(x)} f_{1}(x) \mathcal{A}_{\tau(x)} f_{2}(x),
$$

where $\tau$ is a measurable function from $\mathbb{R}^{n}$ to $[0, \infty)$. For $z \in \mathcal{S}:=\{z \in \mathbb{C}: 0 \leq \operatorname{Re}(z) \leq 1\}$, consider the functions

$$
\frac{1}{l(z)}:=\frac{1-z}{p}+\frac{z}{q}, \quad \frac{1}{l_{i}(z)}:=\frac{1-z}{p_{i}}+\frac{z}{q_{i}}, \quad i=1,2 .
$$

Choose $\theta \in(0,1)$ such that

$$
\frac{1}{l(\theta)}:=\frac{1-\theta}{p}+\frac{\theta}{q}=\frac{1}{\tilde{p}}, \quad \frac{1}{l_{i}(\theta)}:=\frac{1-\theta}{p_{i}}+\frac{\theta}{q_{i}}=\frac{1}{\tilde{p}_{i}}, \quad i=1,2 .
$$

Note that for any linear operator $T$ and a positive number $k \in(0,1)$ satisfying $\frac{k}{p}+\frac{k}{q}<1$ and $k<\tilde{p}$, we can write the following

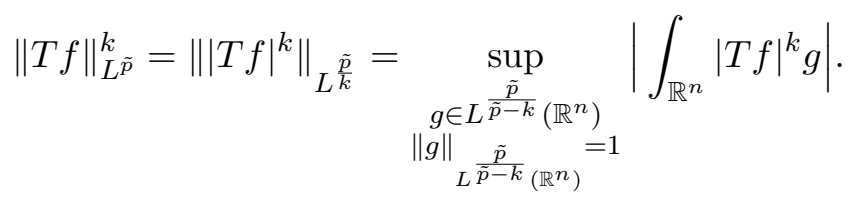

Consider

$$
\begin{aligned}
& \widetilde{v}_{N}(x)=v(x), \quad \text { if } \quad v(x) \leq N \quad \text { and } \quad \widetilde{v}_{N}(x)=N, \quad \text { if } \quad v(x)>N, \\
& \widetilde{w}_{N}(x)=w(x), \quad \text { if } \quad w(x) \leq N \quad \text { and } \quad \widetilde{w}_{N}(x)=N, \quad \text { if } \quad w(x)>N .
\end{aligned}
$$

Let $f_{1}, f_{2}$ be finite simple functions and $g$ be a non-negative finite simple function such that $\left\|f_{i}\right\|_{L^{\tilde{p}_{1}\left(\mathbb{R}^{n}\right)}}=1$, for $i=1,2$ and $\|g\|_{L^{\frac{\tilde{p}}{\tilde{p}}\left(\mathbb{R}^{n}\right)}}=1$.

With the notations introduced as above, consider the following function

$$
\psi(z):=\int_{\mathbb{R}^{n}}\left|\mathcal{A}_{\tau(x)} f_{1, z}(x) \mathcal{A}_{\tau(x)} f_{2, z}(x) \widetilde{v}_{N}^{\frac{z}{q}} \widetilde{w}_{N}^{\frac{1-z}{p}} g^{\frac{\left(1-\frac{k}{l(z)}\right)}{k\left(1-\frac{k}{p}\right)}}\right|^{k} d x,
$$

where

$$
f_{j, z}(x):=\left|f_{j}(x)\right|^{\frac{\tilde{p}_{j}}{l_{j}(z)}} e^{i u_{j}}\left(v_{j}+\epsilon\right)^{\frac{-z}{q_{j}}}\left(w_{j}+\epsilon\right)^{\frac{z-1}{p_{j}}}, \quad j=1,2,
$$


for $z \in \mathcal{S}, \epsilon>0$ and $u_{j} \in[0,2 \pi]$. Note that we have the following expression for $\psi(\theta), \theta \in(0,1)$,

$$
\psi(\theta)=\int_{\mathbb{R}^{n}}\left|\prod_{j=1}^{2} \mathcal{A}_{\tau(x)}\left(f_{j}\left(v_{j}+\epsilon\right)^{-\frac{\theta}{q_{j}}}\left(w_{j}+\epsilon\right)^{\frac{\theta-1}{p_{j}}}\right)(x) \widetilde{v}_{N}^{\frac{\theta}{q}} \widetilde{w}_{N}^{\frac{1-\theta}{p}}\right|^{k} g(x) d x .
$$

For each $x \in \mathbb{R}^{n}$, the functions $\mathcal{A}_{\tau(x)} f_{j, z}(x), \widetilde{v}_{N}^{\frac{z}{q}}(x), \widetilde{w}_{N}^{\frac{1-z}{p}}(x)$ and $g^{\frac{\left(1-\frac{k}{l(z)}\right)}{k\left(1-\frac{k}{p}\right)}}(x)$ are analytic in the domain $\{z \in \mathbb{C}: 0<\operatorname{Re}(z)<1\}$. Therefore the integrand in (9) is a continuous in $z \in \mathcal{S}$ and subharmonic in the domain $\{z \in \mathbb{C}: 0<\operatorname{Re}(z)<1\}$. Also, using the Hölder's inequality with exponents $\frac{p}{k}$ and $\frac{p}{p-k}$, it is easy to see that $\psi$ is a bounded function. Moreover, the Hölder's inequality with exponents $\frac{p}{k}$ and $\frac{p}{p-k}$ and the fact that $\left\|f_{i}\right\|_{L^{\tilde{p}_{i}\left(\mathbb{R}^{n}\right)}}=1, i=1,2$ and $\|g\|_{L \frac{\tilde{p}}{\tilde{p}-k}\left(\mathbb{R}^{n}\right)}=1$, yield that

$$
|\psi(i t)| \leq C_{1}^{k} .
$$

Similarly, using the Hölder's inequality with exponents $\frac{q}{k}$ and $\frac{q}{q-k}$, we get

$$
|\psi(1+i t)| \leq C_{2}^{k}
$$

The constants $C_{1}, C_{2}$ are independent of $\epsilon, N$ and $\tau$.

We invoke the maximum modulus principle for subharmonic functions to deduce that

$$
|\psi(\theta)|=\int_{\mathbb{R}^{n}}\left|\prod_{j=1}^{2} \mathcal{A}_{\tau(x)}\left(f_{j} v_{j, \epsilon}^{-\frac{\theta}{q_{j}}} w_{j, \epsilon}^{\frac{\theta-1}{p_{j}}}\right)(x) \widetilde{v}_{N}^{\frac{\theta}{q}} \widetilde{w}_{N}^{\frac{1-\theta}{p}}\right|^{k} g(x) d x \leq C_{1}^{k(1-\theta)} C_{2}^{k \theta} .
$$

Here we have used the notation $v_{j, \epsilon}=v_{j}+\epsilon$ and $w_{j, \epsilon}=w_{j}+\epsilon$ for $j=1,2$. Therefore, using a duality argument we obtain that

$$
\begin{aligned}
\left(\int_{\mathbb{R}^{n}}\left(\left|\mathcal{A}_{\tau(x)}\left(f_{1} v_{1, \epsilon}^{-\frac{\theta}{q_{1}}} w_{1, \epsilon}^{\frac{\theta-1}{p_{1}}}\right)(x) \mathcal{A}_{\tau(x)}\left(f_{2} v_{2, \epsilon}^{-\frac{\theta}{q_{2}}} w_{2, \epsilon}^{\frac{\theta-1}{p_{2}}}\right)(x)\right| \widetilde{v}_{N}^{\frac{\theta}{q}} \widetilde{w}_{N}^{\frac{1-\theta}{p}}\right)^{\tilde{p}} d x\right)^{\frac{1}{\tilde{p}}} & \\
& \leq C\left(\int_{\mathbb{R}^{n}}\left|f_{1}\right|^{\tilde{p}_{1}}\right)^{\frac{1}{\tilde{\tilde{p}}}}\left(\int_{\mathbb{R}^{n}}\left|f_{2}\right|^{\tilde{p}_{2}}\right)^{\frac{1}{\tilde{\tilde{p}}}} .
\end{aligned}
$$

Since the set of finite simple functions is dense in $L^{s}\left(\mathbb{R}^{n}\right), 1 \leq s<\infty$, we get the estimate above for all $L^{\tilde{p}_{1}}\left(\mathbb{R}^{n}\right)$ functions $f_{1}$ and $f_{2}$ (note that we have assumed $\tilde{p}_{1}=\tilde{p}_{2}$ ). Next, recall that the constants $C_{1}, C_{2}$ are independent of $\epsilon, N$ and $\tau$. Let $\epsilon \rightarrow 0$ and $N \rightarrow \infty$ and replace $f_{i}$ by $f_{i} v_{i}^{\frac{\theta}{q_{i}}} w_{i}^{\frac{1-\theta}{p_{i}}}, i=1,2$, in the above to get that

$$
\begin{aligned}
&\left(\int_{\mathbb{R}^{n}}\left(\left|\mathcal{A}_{\tau(x)}\left(f_{1}\right)(x) \mathcal{A}_{\tau(x)}\left(f_{2}\right)(x)\right| v^{\frac{\theta}{q}} w^{\frac{1-\theta}{p}}\right)^{\tilde{p}} d x\right)^{\frac{1}{\tilde{p}}} \\
& \leq C\left(\int_{\mathbb{R}^{n}}\left|f_{1}\right|^{\tilde{p}_{1}}\left(v_{1}^{\frac{\theta}{q_{1}}} w_{1}^{\frac{1-\theta}{p_{1}}}\right)^{\tilde{p}_{1}}\right)^{\frac{1}{\tilde{p}_{1}}}\left(\int_{\mathbb{R}^{n}}\left|f_{2}\right|^{\tilde{p}_{2}}\left(v_{2}^{\frac{\theta}{q_{2}}} w_{2}^{\frac{1-\theta}{p_{2}}}\right)^{\tilde{p}_{2}}\right)^{\frac{1}{\tilde{p}_{2}}} .
\end{aligned}
$$

Since the constant $C$ is independent of $\tau$, therefore we get the boundedness of the operator $\mathcal{M}_{\text {lac }}$, i.e.

$$
\begin{aligned}
& \left(\int_{\mathbb{R}^{n}}\left(\left|\mathcal{M}_{\operatorname{lac}}\left(f_{1}, f_{2}\right)(x)\right| v^{\frac{\theta}{q}} w^{\frac{1-\theta}{p}}\right)^{\tilde{p}} d x\right)^{\frac{1}{\tilde{p}}} \\
& \quad \leq C\left(\int_{\mathbb{R}^{n}}\left|f_{1}\right|^{\tilde{p}_{1}}\left(v_{1}^{\frac{\theta}{q_{1}}} w_{1}^{\frac{1-\theta}{p_{1}}}\right)^{\tilde{p}_{1}}\right)^{\frac{1}{\tilde{p}}}\left(\int_{\mathbb{R}^{n}}\left|f_{2}\right|^{\tilde{p}_{2}}\left(v_{2}^{\frac{\theta}{q_{2}}} w_{2}^{\frac{1-\theta}{p_{2}}}\right)^{\tilde{p}_{2}}\right)^{\frac{1}{\tilde{p}}} .
\end{aligned}
$$

Step II: We shall use the estimate (10) for radial weights with a suitable choice of exponents to conclude the proof of Theorem 2.4. Indeed, we make the following choice. For $\epsilon>0$, let $p_{1}=p_{2}=\frac{2 n}{2 n-1}+2 \epsilon, r_{1}=r_{2}=\frac{2 n}{2 n-1}+\epsilon$ and $\left(\frac{1}{r_{i}}, \frac{1}{s_{i}}\right) \in L_{n}$. Check that for this choice of $\left(\frac{1}{r_{i}}, \frac{1}{s_{i}}\right)$, $t=\frac{s_{1} s_{2}}{s_{1}+s_{2}-s_{1} s_{2}}>1$ and set $\vec{r}=\left(r_{1}, r_{2}, r_{3}\right)$ with $r_{3}=t$. Let $\vec{w}=\left(|x|^{\alpha^{\prime}},|x|^{\beta^{\prime}}\right) \in A_{\vec{p}, \vec{r}}$ and note that the estimate $(7)$ holds for bilinear $A_{\vec{p}, \vec{r}}$ weights. Next, for $0<\delta<\tilde{p}_{1}-1$, choose $q_{1}=q_{2}=\tilde{p}_{1}-\delta$ and $\vec{v}=\left(|x|^{a},|x|^{b}\right)$ with $1-n \leq a, b<(n-1)\left(\tilde{p}_{1}-\delta-1\right)$. Then we know that the estimate (8) 
holds for $\mathcal{M}_{\text {lac }}$. Therefore, by the previous steps the operator $\mathcal{M}_{\text {lac }}$ satisfies the estimate (10) for the above choice of exponents and we get

$$
\begin{aligned}
\left(\int_{\mathbb{R}^{n}} \mid\right. & \left.\left.\mathcal{M}_{\operatorname{lac}}\left(f_{1}, f_{2}\right)\right|^{\tilde{p}}\left(|x|^{\frac{(a+b) \theta}{\tilde{p}_{1}-\delta}+\frac{\left(\alpha^{\prime}+\beta^{\prime}\right)(1-\theta)(2 n-1)}{2(n+\epsilon(2 n-1))}}\right)^{\tilde{p}}\right)^{\frac{1}{\tilde{p}}} \\
& \leq C\left(\int_{\mathbb{R}^{n}}\left|f_{1}\right|^{\tilde{p}_{1}}\left(|x|^{\frac{a \theta}{\tilde{p}_{1}-\delta}+\frac{\alpha^{\prime}(1-\theta)(2 n-1)}{2 n+2 \epsilon(2 n-1)}}\right)^{\tilde{p}_{1}}\right)^{\frac{1}{\tilde{p}_{1}}}\left(\int_{\mathbb{R}^{n}}\left|f_{2}\right|^{\tilde{p}_{2}}\left(|x|^{\frac{b \theta}{\tilde{p}_{2}-\delta}+\frac{\beta^{\prime}(1-\theta)(2 n-1)}{2 n+2 \epsilon(2 n-1)}}\right)^{\tilde{p}_{2}}\right)^{\frac{1}{\tilde{p}_{2}}}
\end{aligned}
$$

with $\theta \in(0,1)$ and $\frac{(1-\theta)(2 n-1)}{n+\epsilon(2 n-1)}+\frac{2 \theta}{\tilde{p}_{1}-\delta}=\frac{1}{\tilde{p}}=\frac{2}{\tilde{p}_{1}}$.

Now, we show that the exponents of weights in the estimate above may be chosen suitably so that they satisfy the hypothesis of Theorem 2.4. Observe that by Lemma 3.4 we have that $\vec{w}=\left(|x|^{\alpha^{\prime}},|x|^{\beta^{\prime}}\right) \in A_{\vec{p}, \vec{r}}$ implies that

$$
|x|^{\frac{\alpha^{\prime} \theta_{1}(2 n-1)}{2 n+2 \epsilon(2 n-1)}} \in A_{\left(\frac{1-r}{r}\right) \theta_{1}}, \quad|x|^{\frac{\beta^{\prime} \theta_{2}(2 n-1)}{2 n+2 \epsilon(2 n-1)}} \in A_{\left(\frac{1-r}{r}\right) \theta_{2}}, \text { and }|x|^{\frac{\left(\alpha^{\prime}+\beta^{\prime}\right) \delta_{3}(2 n-1)}{2 n+2 \epsilon(2 n-1)}} \in A_{\frac{1-r}{r} \delta_{3}},
$$

where

$$
\frac{1}{\delta_{i}}=\frac{1}{r_{i}}-\frac{1}{p_{i}}, \quad \frac{1}{\theta_{i}}=\frac{1-r}{r}-\frac{1}{\delta_{i}} \quad \text { and } \quad p_{3}=p^{\prime}, \quad i=1,2,3 .
$$

Substituting the values of the various parameters, we obtain

$$
\begin{aligned}
\frac{1-r}{r} & =\frac{(2 n-2)(1+\epsilon(2 n-1))}{2 n+\epsilon(2 n-1)}, \\
\frac{1}{\theta_{i}} & =\frac{\epsilon(2 n-1)(2 n-2)-1}{2 n+\epsilon(2 n-1)}+\frac{2 n-1}{2 n+2 \epsilon(2 n-1)}, \quad \text { for } \quad i=1,2, \\
\frac{1}{\delta_{3}} & =\frac{\epsilon(2 n-1)^{2}}{2 n+\epsilon(2 n-1)}+\frac{2 n-1}{n+\epsilon(2 n-1)}-1 .
\end{aligned}
$$

Since $\epsilon$ can be chosen arbitrarily small, therefore taking $\epsilon \rightarrow 0$ we get $(1-n) \frac{2 n}{2 n-1}<\alpha^{\prime}, \beta^{\prime}<0$ and $(1-n) \frac{2 n}{2 n-1}<\alpha^{\prime}+\beta^{\prime}<0$.

Now taking $\delta \rightarrow \tilde{p}_{1}-1$, we get $\theta=\frac{2 n}{\tilde{p}_{1}}-(2 n-1)$. Since the range of $\alpha^{\prime}$ and $\beta^{\prime}$ is an open set, we get that $\mathcal{M}_{\text {lac }}$ is bounded from $L^{\tilde{p}_{1}}\left(|x|^{\alpha}\right) \times L^{\tilde{p}_{2}}\left(|x|^{\beta}\right)$ to $L^{\tilde{p}}\left(|x|^{\frac{\alpha+\beta}{2}}\right)$ for $\alpha, \beta$ satisfying satisfying

$$
(1-n) \tilde{p}_{1}<\alpha, \beta<0 \quad \text { and } \quad \alpha+\beta>2(1-n)\left(n-(n-1) \tilde{p}_{1}\right) .
$$

Further, using the product-type weighted boundedness of $\mathcal{M}_{\text {lac }}$ for $\tilde{p}_{1}=\tilde{p}_{2}$, we get $\mathcal{M}_{\text {lac }}$ is bounded from $L^{\tilde{p}_{1}}\left(|x|^{a}\right) \times L^{\tilde{p}_{2}}\left(|x|^{b}\right)$ to $L^{\tilde{p}}\left(|x|^{\frac{a+b}{2}}\right)$ for $1-n \leq a, b<(n-1)\left(\tilde{p}_{1}-1\right)$.

This proves the desired result for the operator $\mathcal{M}_{\text {lac }}$.

\section{Comparing Theorem 2.1 With Hölder type Results}

In this section we compare the weighted estimates obtained in Theorem 2.1 with the estimates that can be deduced using Hölder's inequality for both the operator $\mathcal{M}_{\text {lac }}$ and $\mathcal{M}_{\text {full }}$. For $1<p<\infty$, define the sets $\mathcal{L}_{p}:=\left\{w: M_{\text {lac }}\right.$ maps $L^{p}(w)$ to $\left.L^{p}(w)\right\}$ and $\mathcal{F}_{p}:=\{w:$ $M_{\text {full }}$ maps $L^{p}(w)$ to $\left.L^{p}(w)\right\}$. Recall also the definition of $\mathcal{R}_{p}$ in (5) and let $\widetilde{\mathcal{R}}_{p}$ be defined as

$$
\widetilde{\mathcal{R}}_{p}:=\left\{|x|^{b}: 1-n<b<(n-1)(p-1)-1\right\}, \quad n \geq 2 .
$$

In [13], Duoandikoetxea and Vega proved the following weighted estimates for spherical maximal functions with respect to radial weights.

Theorem 5.1. [13] $\mathcal{R}_{p} \subseteq \mathcal{L}_{p}, 1<p<\infty$ and $\widetilde{\mathcal{R}}_{p} \subseteq \mathcal{F}_{p}, \frac{n}{n-1}<p<\infty$.

Let $\frac{1}{\phi_{\text {full }}\left(\frac{1}{r}\right)}$ denote the piecewise linear function on $\left(0, \frac{n-1}{n}\right)$ whose graph connects the points $(0,1),\left(\frac{n^{2}-n}{n^{2}+1}, \frac{n^{2}-n+2}{n^{2}+1}\right)$ and $\left(\frac{n-1}{n}, \frac{n-1}{n}\right)$, defined similarly as (4). Recently, in [20] Lacey proved the weighted estimates for the operators with respect to general weights using sparse domination principle.

Theorem 5.2. [20] The following estimates hold. 


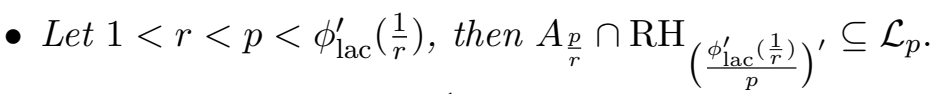

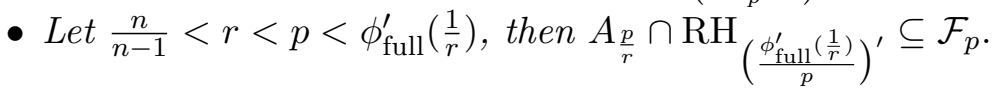

For $\vec{p}=\left(p_{1}, p_{2}, p\right)$ with $\frac{1}{p}=\frac{1}{p_{1}}+\frac{1}{p_{2}}$, define

$$
\mathcal{L}_{\vec{p}}:=\left\{\vec{w}=\left(w_{1}, w_{2}\right): \mathcal{M}_{\text {lac }} \text { maps } L^{p_{1}}\left(w_{1}\right) \times L^{p_{2}}\left(w_{2}\right) \text { to } L^{p}(w)\right\}
$$

and

$$
\mathcal{F}_{\vec{p}}:=\left\{\vec{w}=\left(w_{1}, w_{2}\right): \mathcal{M}_{\text {full }} \text { maps } L^{p_{1}}\left(w_{1}\right) \times L^{p_{2}}\left(w_{2}\right) \text { to } L^{p}(w)\right\} .
$$

In view of Theorems 5.1 and 5.2, Hölder's inequality yields the following weighted estimates for bilinear spherical maximal functions with respect to product type bilinear weights.

Theorem 5.3. The following holds:

- $\prod_{i=1}^{2} A_{\frac{p_{i}}{r_{i}}} \cap \mathrm{RH}{ }_{\left(\frac{\phi_{\text {lac }}^{\prime}\left(\frac{1}{r_{i}}\right)}{p_{i}}\right)^{\prime}} \subseteq \mathcal{L}_{\vec{p}}$ for all $1<r_{i}<p_{i}<\phi_{\text {lac }}^{\prime}\left(\frac{1}{r_{i}}\right)$ and $\prod_{i=1}^{2} \mathcal{R}_{p_{i}} \subseteq \mathcal{L}_{\vec{p}}$, where $p_{i}>1, i=1,2$.

- $\prod_{i=1}^{2} A A_{\frac{p_{i}}{r_{i}}} \cap \mathrm{RH}{ }_{\left(\frac{\phi_{\text {full }}^{\prime}\left(\frac{1}{r_{i}}\right)}{p_{i}}\right)^{\prime}} \subseteq \mathcal{F}_{\vec{p}}$ for all $\frac{n}{n-1}<r_{i}<p_{i}<\phi_{\text {full }}^{\prime}\left(\frac{1}{r_{i}}\right)$ and $\prod_{i=1}^{2} \widetilde{\mathcal{R}}_{p_{i}} \subseteq \mathcal{F}_{\vec{p}}$, where $p_{i}>\frac{n}{n-1}, i=1,2$.

We show that Theorem 2.1 addresses the weighted boundedness of bilinear operators $\mathcal{M}_{\text {lac }}$ and $\mathcal{M}_{\text {full }}$ with respect to bilinear weights that are not of product type as covered by Theorem 5.3 above. Note that it is enough to consider the case of $p_{1}=p_{2}=p$ and point out the difference between the two weighted estimates for power weights.

5.1. The case of full spherical maximal operator $\mathcal{M}_{\text {full }}$. The following result may be deduced from Theorem 2.1.

Corollary 5.4. The operator $\mathcal{M}_{\text {full }}$ is bounded from $L^{p}\left(|x|^{\alpha}\right) \times L^{p}\left(|x|^{\beta}\right)$ to $L^{\frac{p}{2}}\left(|x|^{\frac{\alpha+\beta}{2}}\right)$, for $\alpha, \beta$ satisfying the following conditions:

(i) For $n \geq 2$ and $\frac{n}{n-1}<p \leq \frac{n^{2}+1}{n^{2}-n}$,

$$
\frac{-2 n}{n-1}(p(n-1)-2)<\alpha, \beta<0 \quad \text { with } \quad \frac{-2 n}{n-1}(p(n-1)-2)<\alpha+\beta<0
$$

or, for $n \geq 3$, and $\frac{n}{n-1}<p \leq \frac{n^{2}+1}{n^{2}-n}$

$$
-(n(p+1)-3 p)<\alpha, \beta<n(p-1)-p \quad \text { with } \quad-2(n-p)<\alpha+\beta<2(n(p-1)-p)
$$

or, for $n=2$, and $2<p<\frac{5}{2}$

$$
\frac{-2}{3}(p-2)<\alpha, \beta<\frac{2}{3}(p-2) \quad \text { with } \quad 0<\alpha+\beta<\frac{4}{3}(p-2) .
$$

(ii) For $n \geq 2$ and $\frac{n^{2}+1}{n^{2}-n}<p<\infty$,

$$
-2(n-1)<\alpha, \beta<0 \quad \text { with } \quad-2(n-1)<\alpha+\beta<0 .
$$

(iii) For $n \geq 2$ and $\frac{n^{2}+1}{n^{2}-n}<p \leq \frac{n^{2}+1}{n-1}$,

$\frac{-n}{n+1}(p(n-1)-2)<\alpha, \beta<\frac{n}{n+1}(p(n-1)-2) \quad$ with $\quad 0<\alpha+\beta<\frac{2 n}{n+1}(p(n-1)-2)$ or

$$
\frac{-n}{n^{2}+1}\left((n-1)(n-2) p+n^{2}+1\right)<\alpha, \beta<\frac{n}{n^{2}+1}\left(\left(n^{2}-n\right) p-n^{2}-1\right)
$$

with

$$
\frac{-2 n}{n^{2}+1}\left(n^{2}+1-p(n-1)\right)<\alpha+\beta<\frac{2 n}{n^{2}+1}\left(\left(n^{2}-n\right) p-n^{2}-1\right) .
$$

(iv) For $n \geq 2$ and $\frac{n^{2}+1}{n-1}<p<\infty$,

$$
-n(n-1)<\alpha, \beta<n(n-1) \quad \text { with } \quad 0<\alpha+\beta<2 n(n-1) .
$$


Proof. Proof of (i). Consider $p_{1}=p_{2}=p$ with $\frac{n}{n-1}<p \leq \frac{n^{2}+1}{n^{2}-n}$. Choose $r_{1}=r_{2}=p-\epsilon$, where $0<\epsilon<p-\frac{n}{n-1}$. For this choice of $r_{i}$ we have $\frac{1}{s_{i}}=2-\frac{n+1}{(n-1)(p-\epsilon)}$. Therefore,

$$
\frac{1}{t}=\frac{1}{s_{1}}+\frac{1}{s_{2}}-1=4-\frac{2(n+1)}{(n-1)(p-\epsilon)}-1=\frac{3(n-1)(p-\epsilon)-2(n+1)}{(n-1)(p-\epsilon)} .
$$

Recall that we need to make sure of the condition $t^{\prime}>\frac{p}{2}$. This gives us that $\epsilon<\frac{p[(p+1)(n-1)-(n+1)]}{(n-1)(p+1)}$. Therefore, we have that

$$
0<\epsilon<\min \left\{p-\frac{n}{n-1}, \frac{p[(p+1)(n-1)-(n+1)]}{(n-1)(p+1)}\right\} .
$$

We shall use Lemma 3.4 to get the condition on the exponent of power weights. Following the notation therein, we can write down the parameters $r, \theta_{i}$ and $\delta_{3}$ with the above choice of $r_{i}, s_{i}$ as follows

$$
\begin{aligned}
\frac{1-r}{r} & =\frac{2(n-1)(p-\epsilon)-4}{(n-1)(p-\epsilon)} \\
\frac{1}{\theta_{i}} & =\frac{(2 p+1)(n-1)(p-\epsilon)-p(n+3)}{p(p-\epsilon)(n-1)}, \quad \text { for } \quad i=1,2 \\
\frac{1}{\delta_{3}} & =\frac{2(n-1) p(p-\epsilon)-2 p(n+1)+2(n-1)(p-\epsilon)}{p(p-\epsilon)(n-1)} .
\end{aligned}
$$

We know that $\left(|x|^{\alpha}\right)^{\frac{\theta_{1}}{p}} \in A_{\frac{1-r}{r} \theta_{1}}$ and $\left(|x|^{\frac{\alpha+\beta}{2}}\right)^{\frac{2 \delta_{3}}{p}} \in A_{\frac{1-r}{r} \delta_{3}}$ imply

$$
\frac{-n((2 p+1)(n-1)(p-\epsilon)-p(n+3))}{(n-1)(p-\epsilon)}<\alpha<\frac{n \epsilon}{p-\epsilon}
$$

and

$$
\frac{-n(2(n-1) p(p-\epsilon)-2 p(n+1)+2(n-1)(p-\epsilon))}{(n-1)(p-\epsilon)}<\alpha+\beta<\frac{2 n \epsilon}{p-\epsilon}
$$

Since the conditions above hold for arbitrarily small $\epsilon$, taking $\epsilon \rightarrow 0$, we get that

$$
\frac{-2 n(p(n-1)-2)}{n-1}<\alpha<0 \quad \text { with } \quad \frac{-2 n(p(n-1)-2)}{n-1}<\alpha+\beta<0 .
$$

Observe that due to symmetry of the bilinear operator we get the same condition on $\beta$ as given above for $\alpha$.

This proves the first condition in (i). The proof for the other cases in (i) as well as the other parts (ii)-(iv) may be completed by using the same idea, therefore we skip it.

Now it is easy to compare the exponents of power weights obtained in Corollary 5.4 with that of the Hölder type power weights as given in Theorem 5.3. Recall that in Theorem 5.3 we have that $\mathcal{M}_{\text {full }}$ maps $L^{p}\left(|x|^{a}\right) \times L^{p}\left(|x|^{b}\right)$ to $L^{\frac{p}{2}}\left(|x|^{\frac{a+b}{2}}\right)$ for $1-n<a, b<(n-1)(p-1)-1$. Further, note that this range of exponents $a$ and $b$ is the best possible, except possibly at the point $1-n$, that can be obtained through the classical full spherical maximal function $M_{\text {full }}$ along with the Hölder inequality. On the other hand observe that in Corollary 5.4 we get a significantly better range of $\alpha$ and $\beta$ for which the operator $\mathcal{M}_{\text {full }}$ maps $L^{p}\left(|x|^{\alpha}\right) \times L^{p}\left(|x|^{\beta}\right)$ to $L^{\frac{p}{2}}\left(|x|^{\frac{\alpha+\beta}{2}}\right)$ for $\frac{n}{n-1}<p<\infty$. This is the advantage of our method, that give us improved weighted estimates for the operator $\mathcal{M}_{\text {full }}$ with respect to genuine bilinear weights that are not possible using the Hölder's inequality.

5.2. The case of lacunary spherical maximal operator $\mathcal{M}_{\text {lac }}$. As in the previous case, we can deduce the following weighted estimates operator $\mathcal{M}_{\text {lac }}$ with respect to power weights.

Corollary 5.5. Let $n \geq 2$. The operator $\mathcal{M}_{\text {lac }}$ is bounded from $L^{p}\left(|x|^{\alpha}\right) \times L^{p}\left(|x|^{\beta}\right)$ to $L^{\frac{p}{2}}\left(|x|^{\frac{\alpha+\beta}{2}}\right)$ for $\alpha, \beta$ satisfying the following conditions: 
(i) For $\frac{2 n}{2 n-1}<p \leq \frac{n+1}{n}$,

$$
-2 n(n-1)(p-1)<\alpha, \beta<0 \quad \text { with } \quad-2 n(n-1)(p-1)<\alpha+\beta<0
$$

or

$$
-\left(n-\frac{p}{2}\right)<\alpha, \beta<n(p-1)-\frac{p}{2} \quad \text { with } \quad-n(2-p)<\alpha+\beta<2 n(p-1)-p .
$$

(ii) For $\frac{n+1}{n}<p<\infty$,

$$
-2(n-1)<\alpha, \beta<0 \quad \text { with } \quad-2(n-1)<\alpha+\beta<0 .
$$

(iii) For $\frac{n+1}{n}<p \leq n+1$,

$$
\begin{aligned}
& \frac{-n(n-2) p}{n+1}-n<\alpha, \beta<\frac{n^{2} p}{n+1}-n \quad \text { with } \quad-2 n\left(1-\frac{p}{n+1}\right)<\alpha+\beta<\frac{2 n^{2} p}{n+1}-2 n \\
& \quad \text { or for } 2<p \leq n+1 \\
& \quad-(n-1)(p-1)<\alpha, \beta<(n-1)(p-1) \quad \text { with } 0<\alpha+\beta<2(n-1)(p-1) .
\end{aligned}
$$

(iv) For $n+1 \leq p<\infty$,

$$
-n(n-1)<\alpha, \beta<n(n-1) \quad \text { with } \quad 0<\alpha+\beta<2 n(n-1) .
$$

We skip the proof of the corollary above because it uses similar computations as in the case of Corollary 5.4. Moreover, it can be verified that the range of exponents covered in Corollary 5.5 is better than that given in Theorem 5.3 for the $L^{p}\left(|x|^{\alpha}\right) \times L^{p}\left(|x|^{\beta}\right)$ to $L^{\frac{p}{2}}\left(|x|^{\frac{\alpha+\beta}{2}}\right)$ boundedness of the operator $\mathcal{M}_{\text {lac }}$ for all $1<p<\infty$. As a final remark, observe that part (ii) is not comparable to parts (iii) and (iv) in both corollaries. For example, in the case of $\mathcal{M}_{\text {lac }}$, the individual range of $\alpha, \beta$ in (iv) is better than the individual range of $\alpha, \beta$ in (ii) for $n+1<p<\infty$, but we cannot choose both $\alpha, \beta$ negative due to the condition $0<\alpha+\beta<2 n(n-1)$. However, in (ii) we could do that.

\section{Sparse domination: Proof of Theorem 2.3 (sufficiency)}

In this section we prove the sufficient part of Theorem 2.3. We have exploited the corresponding ideas for the linear case from [20]. As announced, we will proceed proving the sparse domination for characteristic functions and later extend it to general functions. The argument when passing from characteristic functions to general functions is a bit tricky and we use a twostep procedure without which we would obtain an extra condition restricting the result to the Banach range. We follow a unified approach, stating as simultaneously as possible the results for both $\mathcal{M}_{\text {lac }}$ and $\mathcal{M}_{\text {full }}$.

Theorem 6.1. Let $n \geq 2$. For $i=1,2$, let $\left(\frac{1}{r_{i}}, \frac{1}{s_{i}}\right)$ be in the interior of the triangle $L_{n}$ (respectively the trapezium $F_{n}$ ). Then for characteristic functions $f_{1}=\chi_{F_{1}}, f_{2}=\chi_{F_{2}}$ and compactly supported bounded function $h$, where $F_{1}, F_{2}$ are bounded measurable subsets of $\mathbb{R}^{n}$, there exists a sparse collection $\mathcal{S}=\mathcal{S}_{r_{1}, r_{2}, t}$ such that

$$
\left\langle\mathcal{M}\left(f_{1}, f_{2}\right), h\right\rangle \leq C \Lambda_{\mathcal{S}_{r_{1}, r_{2}, t}}\left(f_{1}, f_{2}, h\right),
$$

where $t:=\frac{s_{1} s_{2}}{s_{1}+s_{2}-s_{1} s_{2}}>1$ and $\mathcal{M}:=\mathcal{M}_{\text {lac }}$ (respectively $\left.\mathcal{M}_{\text {full }}\right)$.

For a cube $Q \subset \mathbb{R}^{n}$ with side-length $l_{Q}=2^{q}$, define $\mathcal{A}_{Q} f_{i}(x)=\mathcal{A}_{2^{q-3}}\left(f_{i} \chi_{\frac{1}{3} Q}\right)(x), i=1,2$. Note that $\mathcal{A}_{Q} f_{i}$ is supported in $\frac{2}{3} Q$. The following lemma involving stopping time arguments is the key result in the proof of Theorem 6.1.

Lemma 6.2. Let $n \geq 2$. For $i=1,2$, let $\left(\frac{1}{r_{i}}, \frac{1}{s_{i}}\right)$ be in the interior of the triangle $L_{n}$ (respectively the trapezium $F_{n}$ ), with the additional condition $\frac{1}{s_{1}}+\frac{1}{s_{2}}>1$. Let $f_{1}=\chi_{F_{1}}, f_{2}=\chi_{F_{2}}$, where $F_{1}, F_{2}$ are measurable subsets of $Q_{0}$ and $h$ be a bounded function supported in $Q_{0}$. Let $C_{0}>1$ be a constant and let $\mathcal{D}_{0}$ be a collection of dyadic subcubes of $Q_{0}$ such that

$$
\sup _{Q^{\prime} \in \mathcal{D}_{0}} \sup _{Q: Q^{\prime} \subset Q \subset Q_{0}}\left(\frac{\left\langle f_{i}\right\rangle_{Q, r_{i}}}{\left\langle f_{i}\right\rangle_{Q_{0}, r_{i}}}+\frac{\langle h\rangle_{Q, t}}{\langle h\rangle_{Q_{0}, t}}\right) \leq C_{0}, \text { for } i=1,2,
$$

where $t=\frac{s_{1} s_{2}}{s_{1}+s_{2}-s_{1} s_{2}}>1$. Then, 
(i) If $\left(\frac{1}{r_{i}}, \frac{1}{s_{i}}\right)$ are in the interior of $L_{n}$, with the additional condition $\frac{1}{s_{1}}+\frac{1}{s_{2}}>1$,

$$
\left|\left\langle\sup _{Q \in \mathcal{D}_{0}} \mathcal{A}_{Q} f_{1} \mathcal{A}_{Q} f_{2}, h\right\rangle\right| \lesssim\left|Q_{0}\right|\left\langle f_{1}\right\rangle_{Q_{0}, r_{1}}\left\langle f_{2}\right\rangle_{Q_{0}, r_{2}}\langle h\rangle_{Q_{0}, t}
$$

(ii) If $\left(\frac{1}{r_{i}}, \frac{1}{s_{i}}\right)$ are in the interior of $F_{n}$, with the additional condition $\frac{1}{s_{1}}+\frac{1}{s_{2}}>1$,

$$
\left|\left\langle\sup _{Q \in \mathcal{D}_{0} 2^{q-4} \leq t \leq 2^{q-3}} \sup _{t}\left(f_{1} \chi_{\frac{1}{3} Q}\right)(x) \mathcal{A}_{t}\left(f_{2} \chi_{\frac{1}{3} Q}\right), h\right\rangle\right| \lesssim\left|Q_{0}\right|\left\langle f_{1}\right\rangle_{Q_{0}, r_{1}}\left\langle f_{2}\right\rangle_{Q_{0}, r_{2}}\langle h\rangle_{Q_{0}, t} .
$$

We assume Lemma 6.2 for a moment and complete the proof of Theorems 2.3 and 6.1.

6.1. Proof of Theorem 6.1. We will present the proof for $\mathcal{M}_{\text {lac }}$, and after that we will point out the main differences in the proof for $\mathcal{M}_{\text {full }}$. First note that using standard arguments we can reduce our work to proving analogous results for the dyadic version of the maximal functions under consideration. Indeed, let $f_{1}$ and $f_{2}$ be positive functions with their support contained inside a cube $Q_{0}$. Fix a dyadic lattice $\mathcal{D}$ and consider the maximal function

$$
\mathcal{M}_{\mathcal{D}}\left(f_{1}, f_{2}\right)(x):=\sup _{Q \in \mathcal{D}}\left|\mathcal{A}_{Q} f_{1}(x) \mathcal{A}_{Q} f_{2}(x)\right| .
$$

Since $\operatorname{supp}\left(f_{i}\right) \subset Q_{0}$, we get that $\mathcal{A}_{Q} f_{i}=0$ if $Q \cap Q_{0}=\emptyset$ and also $\mathcal{A}_{Q} f_{i}=0$ for large enough cubes. To justify this, note that we are assuming that $h$ is supported in the cube $Q_{0}$. Therefore, we need to check that for $x \in Q_{0}, \mathcal{A}_{Q} f_{i}(x)=0$, for large enough cube $Q$ (with respect to $Q_{0}$ ). From the definition of $\mathcal{A}_{Q} f_{i}$, we have

$$
\mathcal{A}_{Q} f_{i}(x)=\int_{\mathbb{S}^{n-1}} f_{i}\left(x-2^{q-3} y\right) \chi_{\frac{1}{3} Q}\left(x-2^{q-3} y\right) d \sigma(y) .
$$

Observe that for large enough cube $Q$ with side length $l_{Q}>16 l_{Q_{0}}, x-2^{q-3} y \notin Q_{0}$. Since $\operatorname{supp}\left(f_{i}\right) \subset Q_{0}$, then $\mathcal{A}_{Q} f_{i}(x)=0$.

All in all, in view of the above, it is enough to prove corresponding sparse domination for the bilinear maximal operator

$$
\mathcal{M}_{\mathcal{D} \cap Q_{0}}\left(f_{1}, f_{2}\right)(x)=\sup _{Q \in \mathcal{D} \cap Q_{0}}\left|\mathcal{A}_{Q} f_{1}(x) \mathcal{A}_{Q} f_{2}(x)\right| .
$$

Then, $\left\langle\mathcal{M}_{\text {lac }}\left(f_{1}, f_{2}\right), h\right\rangle$ can be dominated by the sum of finitely many sparse forms. By the definition of supremum, one can find a universal sparse form in the sparse domination.

We proceed to prove the sparse domination result for the operator $\mathcal{M}_{\mathcal{D} \cap Q_{0}}$. Let $C_{0}$ be a constant and $\mathcal{E}_{Q_{0}}$ denote the collection of maximal dyadic subcubes of $Q_{0}$ satisfying

$$
\left\langle f_{1}\right\rangle_{Q, r_{1}}>C_{0}\left\langle f_{1}\right\rangle_{Q_{0}, r_{1}} \quad \text { or } \quad\left\langle f_{2}\right\rangle_{Q, r_{2}}>C_{0}\left\langle f_{2}\right\rangle_{Q_{0}, r_{2}} \quad \text { or } \quad\langle h\rangle_{Q, t}>C_{0}\langle h\rangle_{Q_{0}, t} .
$$

Let $E_{Q_{0}}=\cup_{P \in \mathcal{E}_{Q_{0}}} P$. Note that we can choose $C_{0}>1$ so that $\left|E_{Q_{0}}\right|<\frac{1}{2}\left|Q_{0}\right|$. Writing $F_{Q_{0}}=Q_{0} \backslash E_{Q_{0}}$, we have that $\left|F_{Q_{0}}\right| \geq \frac{1}{2}\left|Q_{0}\right|$.

Next, denote

$$
\mathcal{D}_{0}:=\left\{Q \in \mathcal{D} \cap Q_{0}: Q \cap E_{Q_{0}}=\emptyset\right\}
$$

and observe that for $Q \in \mathcal{D}_{0}$ we get that

$$
\left\langle f_{1}\right\rangle_{Q, r_{1}} \leq C_{0}\left\langle f_{1}\right\rangle_{Q_{0}, r_{1}} \quad \text { and } \quad\left\langle f_{2}\right\rangle_{Q, r_{2}} \leq C_{0}\left\langle f_{2}\right\rangle_{Q_{0}, r_{2}} \quad \text { and } \quad\langle h\rangle_{Q, t} \leq C_{0}\langle h\rangle_{Q_{0}, t} .
$$

For, if (13) holds, then there exists $P \in \mathcal{E}_{Q_{0}}$ such that $P \supset Q$. This will contradict the definition of $\mathcal{D}_{0}$. In a similar way, note that if $Q^{\prime} \in \mathcal{D}_{0}$ and $Q^{\prime} \subset Q \subset Q_{0}$, then we also have (15). These two observations together give us that, for $i=1,2$,

$$
\sup _{Q^{\prime} \in \mathcal{D}_{0} Q: Q^{\prime} \subset Q \subset Q_{0}}\left\langle f_{i}\right\rangle_{Q, r_{i}} \leq C_{0}\left\langle f_{i}\right\rangle_{Q_{0}, r_{i}} \text { and } \sup _{Q^{\prime} \in \mathcal{D}_{0} Q: Q^{\prime} \subset Q \subset Q_{0}} \sup _{Q, t} \leq C_{0}\langle h\rangle_{Q_{0}, t} .
$$

Now we claim, using a standard linearisation argument, that it is enough to prove sparse domination for a suitable linearised form. For, let $\mathcal{Q}$ be the collection of all dyadic subcubes of $Q_{0}$. Given $Q \in \mathcal{Q}$, consider the set

$$
H_{Q}:=\left\{x \in Q: \mathcal{A}_{Q} f_{1}(x) \mathcal{A}_{Q} f_{2}(x) \geq \frac{1}{2} \sup _{P \in \mathcal{Q}} \mathcal{A}_{P} f_{1}(x) \mathcal{A}_{P} f_{2}(x)\right\} .
$$


Note that for any $x \in Q_{0}$, there exists a cube $Q \in \mathcal{Q}$ such that $x \in H_{Q}$. Set $B_{Q}=H_{Q} \backslash$ $\bigcup_{Q^{\prime} \supseteq Q} H_{Q^{\prime}}$. Observe that $\left\{B_{Q}\right\}_{Q \in \mathcal{Q}}$ are pairwise disjoint and $\bigcup_{Q \in \mathcal{Q}} B_{Q}=\bigcup_{Q \in \mathcal{Q}} H_{Q}$. Then

$$
\begin{aligned}
\left\langle\sup _{P \in \mathcal{Q}} \mathcal{A}_{P} f_{1} \mathcal{A}_{P} f_{2}, h\right\rangle & =\sum_{Q \in \mathcal{Q}} \int_{B_{Q}} \sup _{P \in \mathcal{Q}}\left(\mathcal{A}_{P} f_{1}(x) \mathcal{A}_{P} f_{2}(x)\right) h(x) d x \\
& \leq 2 \sum_{Q \in \mathcal{Q}} \int_{B_{Q}} \mathcal{A}_{Q} f_{1}(x) \mathcal{A}_{Q} f_{2}(x) h(x) d x \\
& \leq 2 \sum_{Q \in \mathcal{Q}} \int_{\mathbb{R}^{n}} \mathcal{A}_{Q} f_{1}(x) \mathcal{A}_{Q} f_{2}(x) h(x) \chi_{B_{Q}}(x) d x \\
& =2 \sum_{Q \in \mathcal{Q}}\left\langle\mathcal{A}_{Q} f_{1} \mathcal{A}_{Q} f_{2}, h_{Q}\right\rangle \\
& \leq 2 \sum_{Q \in \mathcal{D} \cap Q_{0}}\left\langle\mathcal{A}_{Q} f_{1} \mathcal{A}_{Q} f_{2}, h \chi_{B_{Q}}\right\rangle
\end{aligned}
$$

where $h_{Q}=h \chi_{B_{Q}}$. The estimate above allows us to work with a linearised form instead of the supremum. Notice that this argument uses the full collection of dyadic subcubes of the given cube $Q_{0}$. So, in particular,

$$
\begin{aligned}
\left|\left\langle\sup _{Q \in \mathcal{D}_{0}} \mathcal{A}_{Q} f_{1} \mathcal{A}_{Q} f_{2}, h\right\rangle\right| \leq\left|\left\langle\sup _{Q \in \mathcal{Q}} \mathcal{A}_{Q} f_{1} \mathcal{A}_{Q} f_{2}, h\right\rangle\right| & \leq\left|2 \sum_{Q \in \mathcal{Q}}\left\langle\mathcal{A}_{Q} f_{1} \mathcal{A}_{Q} f_{2}, h_{Q}\right\rangle\right| \\
& \leq 2\left|\sum_{Q \in \mathcal{D} \cap Q_{0}}\left\langle\mathcal{A}_{Q} f_{1} \mathcal{A}_{Q} f_{2}, h \chi_{B_{Q}}\right\rangle\right| .
\end{aligned}
$$

Therefore, it suffices to prove the sparse domination for

$$
\sum_{Q \in \mathcal{D} \cap Q_{0}}\left\langle\mathcal{A}_{Q} f_{1} \mathcal{A}_{Q} f_{2}, h \chi_{B_{Q}}\right\rangle
$$

Next, observe that for any cube $Q \in \mathcal{D} \cap Q_{0}$ we either have $Q \in \mathcal{D}_{0}$ or $Q \subset P$ for some $P \in \mathcal{E}_{Q_{0}}$. Therefore,

$$
\sum_{Q \in \mathcal{D} \cap Q_{0}}\left\langle\mathcal{A}_{Q} f_{1} \mathcal{A}_{Q} f_{2}, h \chi_{B_{Q}}\right\rangle=\sum_{Q \in \mathcal{D}_{0}}\left\langle\mathcal{A}_{Q} f_{1} \mathcal{A}_{Q} f_{2}, h \chi_{B_{Q}}\right\rangle+\sum_{P \in \mathcal{E}_{Q_{0}}} \sum_{Q \subset P}\left\langle\mathcal{A}_{Q} f_{1} \mathcal{A}_{Q} f_{2}, h \chi_{B_{Q}}\right\rangle .
$$

We would like to remark here that so far we have not required that $f_{1}$ and $f_{2}$ are characteristic functions. Now we invoke Lemma 6.2 to get that

$$
\sum_{Q \in \mathcal{D}_{0}}\left\langle\mathcal{A}_{Q} f_{1} \mathcal{A}_{Q} f_{2}, h \chi_{B_{Q}}\right\rangle \lesssim\left|Q_{0}\right|\left\langle f_{1}\right\rangle_{Q_{0}, r_{1}}\left\langle f_{2}\right\rangle_{Q_{0}, r_{2}}\langle h\rangle_{Q_{0}, t}
$$

Let $\left\{P_{j}\right\}$ be an enumeration of cubes in $\mathcal{E}_{Q_{0}}$. Then we can rewrite the remaining term as

$$
\sum_{P \in \mathcal{E}_{Q_{0}}} \sum_{Q \subset P}\left\langle\mathcal{A}_{Q} f_{1} \mathcal{A}_{Q} f_{2}, h \chi_{B_{Q}}\right\rangle=\sum_{j=1}^{\infty} \sum_{Q \in P_{j} \cap \mathcal{D}}\left\langle\mathcal{A}_{Q} f_{1} \mathcal{A}_{Q} f_{2}, h \chi_{B_{Q}}\right\rangle .
$$

We repeatedly use the estimate above for each $j$ and put all the terms together to get a sparse collection $\mathcal{S}$ so that the following holds

$$
\sum_{Q \in \mathcal{D} \cap Q_{0}}\left\langle\mathcal{A}_{Q} f_{1} \mathcal{A}_{Q} f_{2}, h \chi_{B_{Q}}\right\rangle \lesssim \sum_{S \in \mathcal{S}}|S|\left\langle f_{1}\right\rangle_{S, r_{1}}\left\langle f_{2}\right\rangle_{S, r_{2}}\langle h\rangle_{S, t} .
$$

This completes the proof of Theorem 6.1 for $\mathcal{M}_{\text {lac }}$.

In order to prove the corresponding results for the operator $\mathcal{M}_{\text {full }}$, we require a bilinear analogue of local spherical maximal functions. It is defined as follows

$$
\widetilde{\mathcal{M}}\left(f_{1}, f_{2}\right)(x):=\sup _{t \in[1,2]} \mathcal{A}_{t} f_{1}(x) \mathcal{A}_{t} f_{2}(x) .
$$


Again standard arguments reduce the task to consider a dyadic version with the maximal function

$$
\sup _{Q \in \mathcal{D} \cap Q_{0}}\left|\widetilde{\mathcal{M}}_{Q}\left(f_{1}, f_{2}\right)(x)\right|,
$$

where

$$
\widetilde{\mathcal{M}}_{Q}\left(f_{1}, f_{2}\right)(x):=\sup _{2^{q-4} \leq t \leq 2^{q-3}} \mathcal{A}_{t}\left(f_{1} \chi_{\frac{1}{3} Q}\right)(x) \mathcal{A}_{t}\left(f_{2} \chi_{\frac{1}{3} Q}\right)(x) .
$$

Note that a linearisation trick as earlier tells us that it suffices to replace the supremum (19) with the form

$$
\left|\sum_{Q \in \mathcal{D}_{0}}\left\langle\widetilde{\mathcal{M}}_{Q}\left(f_{1}, f_{2}\right), h_{Q}\right\rangle\right|
$$

with $h_{Q}=h \chi_{B_{Q}}$ and $B_{Q}=E_{Q} \backslash \bigcup_{Q^{\prime} \supset Q} E_{Q^{\prime}}$, where

$$
E_{Q}=\left\{x \in Q \in \mathcal{Q}: \widetilde{\mathcal{M}}_{Q}\left(f_{1}, f_{2}\right)(x) \geq \frac{1}{2} \sup _{P \in \mathcal{Q}} \widetilde{\mathcal{M}}_{P}\left(f_{1}, f_{2}\right)(x)\right\} .
$$

The remaining part of the proof can be completed following the lacunary case.

6.2. Proof of Theorem 2.3. We will make use of Theorem 6.1 in proving Theorem 2.3. The proof is unified in both lacunary and full cases.

Step I: Let $f_{1}$ and $h$ be non-negative compactly supported bounded functions with support in the cube $Q_{0}$ and $f_{2}=\chi_{F_{2}}$, where $F_{2} \subset Q_{0}$. We use the same argument as in the proof of Theorem 6.1 up to the estimate (17) with the same notation and here $\mathcal{D}_{0}$ is defined with respect to $f_{1}, \chi_{F_{2}}$ and $h$. In fact, it is enough to prove an analogue of estimate (18) for the setting under consideration, i.e., we need to show that

$$
\sum_{Q \in \mathcal{D}_{0}}\left\langle\mathcal{A}_{Q} f_{1} \mathcal{A}_{Q} \chi_{F_{2}}, h_{Q}\right\rangle \lesssim\left|Q_{0}\right|\left\langle f_{1}\right\rangle_{Q_{0}, \rho_{1}}\left\langle\chi_{F_{2}}\right\rangle_{Q_{0}, r_{2}}\langle h\rangle_{Q_{0}, t}
$$

where $\rho_{1}>r_{1}$.

In order to use Theorem 6.1, we first need to decompose the function $f_{1}$ into suitable characteristic functions. Consider $E_{m}=\left\{x \in Q_{0}: 2^{m} \leq f_{1}(x) \leq 2^{m+1}\right\}$. Then there exists $m_{0}>1$ such that $E_{m}=\emptyset$ for all $m>m_{0}$. Denote $f_{1}^{m}=f_{1} \chi_{E_{m}}$. Thus, we use Theorem 6.1 for each pair of characteristic functions $\chi_{E_{m}}$ and $\chi_{F_{2}}$ and obtain the sparse domination for the functions $f_{1}^{m}$ and $\chi_{F_{2}}$ as follows

$$
\begin{aligned}
\sum_{Q \in \mathcal{D}_{0}}\left\langle\mathcal{A}_{Q} f_{1}^{m} \mathcal{A}_{Q} \chi_{F_{2}}, h_{Q}\right\rangle & \leq 2^{m+1} \sum_{Q \in \mathcal{D}_{0}}\left\langle\mathcal{A}_{Q} \chi_{E_{m}} \mathcal{A}_{Q} \chi_{F_{2}}, h_{Q}\right\rangle \\
& \lesssim 2^{m+1} \sum_{Q \in \mathcal{D} \cap Q_{0}}\left\langle\mathcal{A}_{Q} \chi_{E_{m}} \mathcal{A}_{Q} \chi_{F_{2}}, h_{Q} \chi_{F_{Q_{0}}}\right\rangle \\
& \lesssim 2^{m+1} \sum_{S \in \mathcal{S}_{m}}|S|\left\langle\chi_{E_{m}}\right\rangle_{S, r_{1}}\left\langle\chi_{F_{2}}\right\rangle_{S, r_{2}}\left\langle h \chi_{F_{Q_{0}}}\right\rangle_{S, t}
\end{aligned}
$$

where $\mathcal{S}_{m}$ is the sparse family corresponding to characteristic functions $\chi_{E_{m}}$ and $\chi_{F_{2}}$.

Next, using the stopping time condition on the functions $h$ and $f_{2}=\chi_{F_{2}}$ as given in (16), we get

$$
\sum_{S \in \mathcal{S}_{m}}|S|\left\langle\chi_{E_{m}}\right\rangle_{S, r_{1}}\left\langle\chi_{F_{2}}\right\rangle_{S, r_{2}}\left\langle h \chi_{F_{Q_{0}}}\right\rangle_{S, t} \lesssim\langle h\rangle_{Q_{0}, t}\left\langle\chi_{F_{2}}\right\rangle_{Q_{0}, r_{2}} \sum_{S \in \mathcal{S}_{m}}|S|\left\langle\chi_{E_{m}}\right\rangle_{S, r_{1}}
$$

Choose $\widetilde{\rho_{1}}>r_{1}$ and consider ${\widetilde{\rho_{1}}}^{\prime}>1$ such that $\frac{1}{\widetilde{\rho_{1}}}+\frac{1}{{\widetilde{\rho_{1}}}^{\prime}}=1$. Now, using an easy consequence of the Carleson embedding theorem (see [21]) we get that

$$
\begin{aligned}
\sum_{S \in \mathcal{S}_{m}}|S|\left\langle\chi_{E_{m}}\right\rangle_{S, r_{1}} & =\sum_{S \in \mathcal{S}_{m}}|S|^{\frac{1}{\tilde{\rho}_{1}}}\left\langle\chi_{E_{m}}\right\rangle_{S, r_{1}}|S|^{\frac{1}{\widetilde{\rho}_{1}^{\prime}}} \\
& \leq\left(\sum_{S \in \mathcal{S}_{m}}|S|\left\langle\chi_{E_{m}}\right\rangle_{S, r_{1}}^{\widetilde{\rho}_{1}}\right)^{\frac{1}{\tilde{\rho}_{1}}}\left(\sum_{S \in \mathcal{S}_{m}}|S|\right)^{\frac{1}{\tilde{\rho}_{1}^{\prime}}} \\
& \leq\left\langle\chi_{E_{m}}\right\rangle_{Q_{0}, \widetilde{\rho}_{1}}\left|Q_{0}\right| .
\end{aligned}
$$


Finally, using [2, Lemma 4.6 and Lemma 4.7], we obtain

$$
\left|\left\langle\sup _{Q \in \mathcal{D}_{0}} \mathcal{A}_{Q} f_{1} \mathcal{A}_{Q} \chi_{F_{2}}, h_{Q}\right\rangle\right| \lesssim\left|Q_{0}\right|\left\langle f_{1}\right\rangle_{Q_{0}, \rho_{1}}\left\langle\chi_{F_{2}}\right\rangle_{Q_{0}, r_{2}}\langle h\rangle_{Q_{0}, t}
$$

where $\rho_{1}>\widetilde{\rho_{1}}$.

Note that we can decompose $\sum_{Q \in \mathcal{D} \cap Q_{0}}\left\langle\mathcal{A}_{Q} f_{1} \mathcal{A}_{Q} \chi_{F_{2}}, h \chi_{B_{Q}}\right\rangle$ as

$$
\sum_{Q \in \mathcal{D} \cap Q_{0}}\left\langle\mathcal{A}_{Q} f_{1} \mathcal{A}_{Q} \chi_{F_{2}}, h \chi_{B_{Q}}\right\rangle=\sum_{Q \in \mathcal{D}_{0}}\left\langle\mathcal{A}_{Q} f_{1} \mathcal{A}_{Q} \chi_{F_{2}}, h \chi_{B_{Q}}\right\rangle+\sum_{P \in \mathcal{E}_{Q_{0}}} \sum_{Q \subset P}\left\langle\mathcal{A}_{Q} f_{1} \mathcal{A}_{Q} \chi_{F_{2}}, h \chi_{B_{Q}}\right\rangle .
$$

Now from (20) we get that

$$
\sum_{Q \in \mathcal{D}_{0}}\left\langle\mathcal{A}_{Q} f_{1} \mathcal{A}_{Q} \chi_{F_{2}}, h \chi_{B_{Q}}\right\rangle \lesssim\left|Q_{0}\right|\left\langle f_{1}\right\rangle_{Q_{0}, \rho_{1}}\left\langle\chi_{F_{2}}\right\rangle_{Q_{0}, r_{2}}\langle h\rangle_{Q_{0}, t}
$$

Let $\left\{P_{j}\right\}$ be an enumeration of cubes in $\mathcal{E}_{Q_{0}}$ defined with $f_{1}, \chi_{F_{2}}$ and $h$. Then we can rewrite the remaining term as

$$
\sum_{P \in \mathcal{E}_{Q_{0}}} \sum_{Q \subset P}\left\langle\mathcal{A}_{Q} f_{1} \mathcal{A}_{Q} \chi_{F_{2}}, h \chi_{B_{Q}}\right\rangle=\sum_{j=1}^{\infty} \sum_{Q \in P_{j} \cap \mathcal{D}}\left\langle\mathcal{A}_{Q} f_{1} \mathcal{A}_{Q} \chi_{F_{2}}, h \chi_{B_{Q}}\right\rangle .
$$

We repeatedly use the estimate above for each $j$ and put all the terms together to get a sparse collection $\mathcal{S}$ so that the following holds

$$
\sum_{Q \in \mathcal{D} \cap Q_{0}}\left\langle\mathcal{A}_{Q} f_{1} \mathcal{A}_{Q} \chi_{F_{2}}, h \chi_{B_{Q}}\right\rangle \lesssim \sum_{S \in \mathcal{S}}|S|\left\langle f_{1}\right\rangle_{S, \rho_{1}}\left\langle\chi_{F_{2}}\right\rangle_{S, r_{2}}\langle h\rangle_{S, t}
$$

Step II: Now consider $f_{1}, f_{2}$ and $h$ are non-negative compactly supported bounded function with support in the cube $Q_{0}$. We use the same argument as in the proof of Theorem $6.1 \mathrm{up}$ to the estimate $(17)$ with the same notation and $\mathcal{D}_{0}$ is defined with respect to $f_{1}, f_{2}$ and $h$. It suffices to prove an analogue of estimate (18) for the setting under consideration, i.e., we need to show that

$$
\sum_{Q \in \mathcal{D}_{0}}\left\langle\mathcal{A}_{Q} f_{1} \mathcal{A}_{Q} f_{2}, h_{Q}\right\rangle \lesssim\left|Q_{0}\right|\left\langle f_{1}\right\rangle_{Q_{0}, \rho_{1}}\left\langle f_{2}\right\rangle_{Q_{0}, \rho_{2}}\langle h\rangle_{Q_{0}, t}
$$

where $\rho_{1}>r_{1}$ and $\rho_{2}>r_{2}$.

Note that we already have an analogue of estimate (18) for general compactly supported bounded function $f_{1}$ and $f_{2}=\chi_{F_{2}}$ a characteristic function in (20). Therefore, in order to prove the required estimate for general compactly supported bounded functions $f_{1}$ and $f_{2}$ we decompose the function $f_{2}$ such that $f_{2}=\sum_{n=1}^{n_{0}} f_{2}^{n}$ with $f_{2}^{n}=f_{2} \chi_{F_{n}}$, where $F_{n}=\left\{x \in Q_{0}\right.$ : $\left.2^{n} \leq f_{2}(x) \leq 2^{n+1}\right\}$ and $F_{n}=\emptyset$ for $n>n_{0}$. From this point onward the proof follows in a similar fashion as in Step I. Finally, we get the following estimate

$$
\left|\left\langle\sup _{Q \in \mathcal{D}_{0}} \mathcal{A}_{Q} f_{1} \mathcal{A}_{Q} f_{2}, h_{Q}\right\rangle\right| \lesssim\left|Q_{0}\right|\left\langle f_{1}\right\rangle_{Q_{0}, \rho_{1}}\left\langle f_{2}\right\rangle_{Q_{0}, \rho_{2}}\langle h\rangle_{Q_{0}, t}
$$

where $\rho_{1}>r_{1}$ and $\rho_{2}>r_{2}$.

Remark 6.3. Observe that the argument in which we freeze one of the characteristic functions when passing from characteristic functions to general functions is crucial. Dealing with both characteristic functions simultaneously yields the extra condition $\frac{1}{r_{1}}+\frac{1}{r_{2}}<1$, restricting the result to the Banach range.

6.3. Proof of Lemma 6.2. Finally we provide the proof of Lemma 6.2. This is the most technical and tedious part of the paper. We will begin by giving the proof in the lacunary case, and after that we will sketch the significantly different parts in case of $\mathcal{M}_{\text {full }}$.

First note that one can use the same linearisation trick as in the proof of Theorem 6.1. This would mean that it is enough to prove the following estimate

$$
\sum_{Q \in \mathcal{D}_{0}}\left\langle\mathcal{A}_{Q} f_{1} \mathcal{A}_{Q} f_{2}, h_{Q}\right\rangle \lesssim\left|Q_{0}\right|\left\langle f_{1}\right\rangle_{Q_{0}, r_{1}}\left\langle f_{2}\right\rangle_{Q_{0}, r_{2}}\langle h\rangle_{Q_{0}, t}
$$


where $\mathcal{A}_{Q} f_{i}(x)=\mathcal{A}_{2^{q-3}}\left(f_{i} \chi_{\frac{1}{3} Q}\right)(x)$, and so

$$
\mathcal{A}_{Q} f_{i}(x)=\int_{\mathbb{S}^{n-1}} f_{i}\left(x-2^{q-3} y\right) \chi_{\frac{1}{3} Q}\left(x-2^{q-3} y\right) d \sigma(y) .
$$

Since $l_{Q}=2^{q}$, we see that for $y \in \mathbb{S}^{n-1}$ we have $\frac{1}{3} Q+2^{q-3} y \subset \frac{2}{3} Q$ and hence $\operatorname{supp}\left(\mathcal{A}_{Q} f_{i}\right) \subset \frac{2}{3} Q$. Now define

$$
\widetilde{\mathcal{A}}_{Q} f_{i}(x)=\mathcal{A}_{2^{q-3}}\left(f_{i} \chi_{\frac{1}{2} Q}\right)(x) .
$$

Observe that $\operatorname{supp}\left(\widetilde{\mathcal{A}}_{Q} f_{i}\right) \subset Q$ and since $f_{i}$ 's are non-negative functions, therefore

$$
\begin{aligned}
\mathcal{A}_{Q} f_{i}(x)=\int_{\mathbb{S}^{n-1}} f_{i}\left(x-2^{q-3} y\right) \chi_{\frac{1}{3} Q}( & \left.-2^{q-3} y\right) d \sigma(y) \\
\leq & \int_{\mathbb{S}^{n-1}} f_{i}\left(x-2^{q-3} y\right) \chi_{\frac{1}{2} Q}\left(x-2^{q-3} y\right) d \sigma(y)=\widetilde{\mathcal{A}}_{Q} f_{i}(x),
\end{aligned}
$$

for a.e. $x$ and $i=1,2$. In view of these observations, it is enough to prove that

$$
\sum_{Q \in \mathcal{D}_{0}}\left\langle\widetilde{\mathcal{A}}_{Q} f_{1} \widetilde{\mathcal{A}}_{Q} f_{2}, h_{Q}\right\rangle \lesssim\left|Q_{0}\right|\left\langle f_{1}\right\rangle_{Q_{0}, r_{1}}\left\langle f_{2}\right\rangle_{Q_{0}, r_{2}}\langle h\rangle_{Q_{0}, t}
$$

The important observation here is that with this localization we will be able to separately distinguish the dyadic (bad) cubes in which the cancellation property can be applied. Here we have used the same notation as in Theorem 6.1. For $i=1,2$, let

$$
\gamma_{f_{i}}=\left\{\text { collection of maximal dyadic subcubes } P \subset Q_{0}:\left\langle f_{i}\right\rangle_{P, r_{i}}>2 C_{0}\left\langle f_{i}\right\rangle_{Q_{0}, r_{i}}\right\} .
$$

Applying the Calderón-Zygmund decomposition to each $f_{i}$ at the height $\alpha_{i}=2 C_{0}\left\langle f_{i}\right\rangle_{Q_{0}, r_{i}}$, $i=1,2$, we can decompose

$$
f_{i}=b_{i}+g_{i}
$$

where $\left\|g_{i}\right\|_{L^{\infty}} \lesssim\left\langle f_{i}\right\rangle_{Q_{0}, r_{i}}$ and

$$
b_{i}=\sum_{P \in \gamma_{f_{i}}}\left(f_{i}-\left\langle f_{i}\right\rangle_{P}\right) \chi_{P}=\sum_{k=-\infty}^{q_{0}-1} \sum_{P \in B_{i}(k)}\left(f_{i}-\left\langle f_{i}\right\rangle_{P}\right) \chi_{P}=: \sum_{k=-\infty}^{q_{0}-1} B_{i, k},
$$

with $l_{Q_{0}}=2^{q_{0}}$ and $B_{i}(k)=\left\{P \in \gamma_{f_{i}}: l_{P}=2^{k}\right\}$. Now,

$$
\begin{aligned}
\left|\sum_{Q \in \mathcal{D}_{0}}\left\langle\widetilde{\mathcal{A}}_{Q} f_{1} \widetilde{\mathcal{A}}_{Q} f_{2}, h_{Q}\right\rangle\right| \leq \mid & \left|\sum_{Q \in \mathcal{D}_{0}}\left\langle\widetilde{\mathcal{A}}_{Q} g_{1} \widetilde{\mathcal{A}}_{Q} g_{2}, h_{Q}\right\rangle\right|+\left|\sum_{Q \in \mathcal{D}_{0}}\left\langle\widetilde{\mathcal{A}}_{Q} g_{1} \widetilde{\mathcal{A}}_{Q} b_{2}, h_{Q}\right\rangle\right| \\
& +\left|\sum_{Q \in \mathcal{D}_{0}}\left\langle\widetilde{\mathcal{A}}_{Q} b_{1} \widetilde{\mathcal{A}}_{Q} g_{2}, h_{Q}\right\rangle\right|+\left|\sum_{Q \in \mathcal{D}_{0}}\left\langle\widetilde{\mathcal{A}}_{Q} b_{1} \widetilde{\mathcal{A}}_{Q} b_{2}, h_{Q}\right\rangle\right| \\
= & : G G+G B+B G+B B .
\end{aligned}
$$

We estimate all the four parts separately. Note that in view of the symmetry in $G B$ and $B G$ parts, it is enough to estimate one of them.

Estimate for $G G$ (both functions good) part. Using the fact that $t>1$, we have

$$
\begin{aligned}
G G \leq \sum_{Q \in \mathcal{D}_{0}}\left\|\widetilde{\mathcal{A}}_{Q} g_{1} \widetilde{\mathcal{A}}_{Q} g_{2}\right\|_{L^{\infty}}\left\|h_{Q}\right\|_{L^{1}} & \lesssim\left\langle f_{1}\right\rangle_{Q_{0}, r_{1}}\left\langle f_{2}\right\rangle_{Q_{0}, r_{2}} \sum_{Q \in \mathcal{D}_{0}} \int\left|h(x) \chi_{B_{Q}}(x)\right| d x \\
& \lesssim\left\langle f_{1}\right\rangle_{Q_{0}, r_{1}}\left\langle f_{2}\right\rangle_{Q_{0}, r_{2}}\langle h\rangle_{Q_{0}}\left|Q_{0}\right| \\
& \lesssim\left\langle f_{1}\right\rangle_{Q_{0}, r_{1}}\left\langle f_{2}\right\rangle_{Q_{0}, r_{2}}\langle h\rangle_{Q_{0}, t}\left|Q_{0}\right| .
\end{aligned}
$$

Estimate for $B G$ (one function bad and one function good) part. Arguing with a similar argument as in the proof of Theorem 6.1 in our paper we note that, for all $Q \in \mathcal{D}_{0}$ and $P \in \gamma_{f_{1}}$, if $P \cap Q \neq \emptyset$, then $P \subsetneq Q$, by the stopping argument. Therefore, for any $Q \in \mathcal{D}_{0}$ with $l_{Q}=2^{q}$, we have

$$
\left\langle\widetilde{\mathcal{A}}_{Q} b_{1} \widetilde{\mathcal{A}}_{Q} g_{2}, h_{Q}\right\rangle=\sum_{k<q}\left\langle\widetilde{\mathcal{A}}_{Q} B_{1, k} \widetilde{\mathcal{A}}_{Q} g_{2}, h_{Q}\right\rangle=\sum_{k=1}^{\infty}\left\langle\widetilde{\mathcal{A}}_{Q} B_{1, q-k} \widetilde{\mathcal{A}}_{Q} g_{2}, h_{Q}\right\rangle
$$


Thus,

$$
\left|\sum_{Q \in \mathcal{D}_{0}}\left\langle\widetilde{\mathcal{A}}_{Q} b_{1} \widetilde{\mathcal{A}}_{Q} g_{2}, h_{Q}\right\rangle\right|=\left|\sum_{k=1}^{\infty} \sum_{Q \in \mathcal{D}_{0}}\left\langle\widetilde{\mathcal{A}}_{Q} B_{1, q-k} \widetilde{\mathcal{A}}_{Q} g_{2}, h_{Q}\right\rangle\right| .
$$

Note that $\left(\widetilde{\mathcal{A}}_{Q}\right)^{*} f(x)=\chi_{\frac{1}{2} Q}(x) \mathcal{A}_{2^{q-3}} f(x)$. Indeed, for compactly supported bounded functions $f$ and $g$, we have

$$
\begin{aligned}
\left\langle\widetilde{\mathcal{A}}_{Q} f, g\right\rangle & =\int_{\mathbb{R}^{n}} \widetilde{\mathcal{A}}_{Q} f(x) g(x) d x \\
& =\int_{\mathbb{R}^{n}}\left(\int_{\mathbb{S}^{n-1}} f \chi_{\frac{1}{2} Q}\left(x-2^{q-3} y\right) d \sigma(y)\right) g(x) d x \\
& =\int_{\mathbb{R}^{n}} f(x) \chi_{\frac{1}{2} Q}(x)\left(\int_{\mathbb{S}^{n-1}} g\left(x+2^{q-3} y\right) d \sigma(y)\right) d x \\
& =:\left\langle f,\left(\widetilde{\mathcal{A}}_{Q}\right)^{*} g\right\rangle
\end{aligned}
$$

where

$$
\left(\widetilde{\mathcal{A}}_{Q}\right)^{*} g(x)=\chi_{\frac{1}{2} Q}(x) \mathcal{A}_{2^{q-3}} g(x)
$$

Hence,

$$
\begin{aligned}
B G & =\left|\sum_{Q \in \mathcal{D}_{0}} \int b_{1}(x)\left(\widetilde{\mathcal{A}}_{Q}\right)^{*}\left(\widetilde{\mathcal{A}}_{Q} g_{2} \cdot h_{Q}\right)(x) d x\right| \\
& =\left|\sum_{Q \in \mathcal{D}_{0}} \sum_{k=1}^{\infty} \sum_{P \in B_{1}(q-k)} \int_{P} B_{1, q-k}(x)\left(\widetilde{\mathcal{A}}_{Q}\right)^{*}\left(\widetilde{\mathcal{A}}_{Q} g_{2} \cdot h_{Q}\right)(x) d x\right| \\
& =\left|\sum_{Q \in \mathcal{D}_{0}} \sum_{k=1}^{\infty} \sum_{P \in B_{1}(q-k)} \int_{P} B_{1, q-k}(x) \chi_{\frac{1}{2} Q}(x) \mathcal{A}_{2^{q-3}}\left(\widetilde{\mathcal{A}}_{Q} g_{2} \cdot h_{Q}\right)(x) d x\right| .
\end{aligned}
$$

Now, observe that in the above line only those $P$ will survive for which $P \cap \frac{1}{2} Q \neq \emptyset$. Further, we can write

$$
\left|\sum_{Q \in \mathcal{D}_{0}} \sum_{k=1}^{\infty} \sum_{P \in B_{1}(q-k)} \int_{P} B_{1, q-k}(x) \chi_{\frac{1}{2} Q}(x) \mathcal{A}_{2^{q-3}}\left(\widetilde{\mathcal{A}}_{Q} g_{2} \cdot h_{Q}\right)(x) d x\right| \leq I+I I,
$$

where

$$
I:=\left|\sum_{Q \in \mathcal{D}_{0}} \sum_{k=1}^{3} \sum_{P \in B_{1}(q-k)} \int_{P} B_{1, q-k}(x) \chi_{\frac{1}{2} Q}(x) \mathcal{A}_{2^{q-3}}\left(\widetilde{\mathcal{A}}_{Q} g_{2} \cdot h_{Q}\right)(x) d x\right|
$$

and

$$
I I:=\left|\sum_{Q \in \mathcal{D}_{0}} \sum_{k=4}^{\infty} \sum_{P \in B_{1}(q-k)} \int_{P} B_{1, q-k}(x) \chi_{\frac{1}{2} Q}(x) \mathcal{A}_{2^{q-3}}\left(\widetilde{\mathcal{A}}_{Q} g_{2} \cdot h_{Q}\right)(x) d x\right| .
$$

Here we have separated subcubes of $k$ th generation with $k \geq 4$ in part $I I$ so that we can use [20, Lemma 2.3]. For the part $I$ we do not need to use the cancellation condition on $B_{1, q-k}$ as there are only finitely many terms with respect to $k$ and the sizes of subcubes $P$ in these generations are comparable with the size of original cube $Q$ with an absolute constant.

Recall that if $P \cap Q \neq \emptyset$, then $P \subsetneq Q$ (by stopping time argument). Therefore, all the $P$ 's are dyadic subcubes of $Q$. Also, observe that all the dyadic subcubes of $Q$ of second and higher generations which intersect (except with edges) with $\frac{1}{2} Q$, are contained in $\frac{1}{2} Q$. In fact, all the second generation subcubes of $Q$ which are around the center of $Q$, are contained in $\frac{1}{2} Q$, and the other second generation subcubes of $Q$ along the boundary of $Q$ do not intersect with $\frac{1}{2} Q$, i.e, for any $P \in B_{1}(q-k), k \geq 2$, with $P \cap \frac{1}{2} Q \neq \emptyset$, implies $P \subset \frac{1}{2} Q$. In particular, this property will be used in estimating part $I I$ for all $k \geq 4$. 
Now, we first deal with part $I$, in which we cannot use the cancellation property (since the dyadic cubes of first generation $P$ are all intersecting $\frac{1}{2} Q$, but $P \not \subset \frac{1}{2} Q$ ). Nevertheless, we will not need the decay to conclude the estimate in this case ${ }^{2}$ :

$$
\begin{aligned}
I & :=\left|\sum_{Q \in \mathcal{D}_{0}} \sum_{k=1}^{3} \sum_{P \in B_{1}(q-k)} \int_{P} B_{1, q-k}(x) \chi_{\frac{1}{2} Q}(x) \mathcal{A}_{2^{q-3}}\left(\widetilde{\mathcal{A}}_{Q} g_{2} \cdot h_{Q}\right)(x) d x\right| \\
& =\left|\sum_{k=1}^{3} \sum_{Q \in \mathcal{D}_{0}} \int_{\mathbb{P}_{k}} B_{1, q-k}(x)\left(\widetilde{\mathcal{A}}_{Q}\right)^{*}\left(\widetilde{\mathcal{A}}_{Q} g_{2} \cdot h_{Q}\right)(x) d x\right|,
\end{aligned}
$$

where $\bigcup_{P \in B_{1}(q-k)} P=\mathbb{P}_{k}$. Since, $P \in B_{1}(q-k)$ are disjoint subcubes of $Q$, we get $\mathbb{P}_{k} \subseteq Q$. Now,

$$
\begin{aligned}
I & =\left|\sum_{k=1}^{3} \sum_{Q \in \mathcal{D}_{0}} \int_{\mathbb{R}^{n}} \widetilde{\mathcal{A}}_{Q}\left(B_{1, q-k} \chi_{\mathbb{P}_{k}}\right)(x) \widetilde{\mathcal{A}}_{Q} g_{2}(x) h_{Q}(x) d x\right| \\
& \leq \sum_{k=1}^{3} \sum_{Q \in \mathcal{D}_{0}}\left\|\widetilde{\mathcal{A}}_{Q}\left(B_{1, q-k} \chi_{\mathbb{P}_{k}}\right)\right\|_{L^{s_{1}}}\left\|\widetilde{\mathcal{A}}_{Q} g_{2} \cdot h_{Q}\right\|_{L^{s_{1}}} \\
& \lesssim \sum_{k=1}^{3} \sum_{Q \in \mathcal{D}_{0}}|Q|\left\langle B_{1, q-k}\right\rangle_{Q, r_{1}}\left\langle\widetilde{\mathcal{A}}_{Q} g_{2} \cdot h_{Q}\right\rangle_{Q, s_{1}} .
\end{aligned}
$$

In the second last line we have applied Hölder's inequality with respect to $s_{1}^{\prime}$ and $s_{1}$, where $\frac{1}{s_{1}}+\frac{1}{s_{1}^{\prime}}=1$ and in the last line we have applied $L^{r_{1}} \rightarrow L^{s_{1}^{\prime}}$ boundedness of the averaging operator $\mathcal{A}_{2^{q-3}}$, for $\left(\frac{1}{r_{1}}, \frac{1}{s_{1}^{\prime}}\right)$ belonging to the interior of $L_{n}^{\prime}$.

Let us now estimate the part $I I$. The key point here is that, for small enough cubes, we first write the dual and then use the cancellation:

$$
\begin{aligned}
I I:= & \left|\sum_{Q \in \mathcal{D}_{0}} \sum_{k=4}^{\infty} \sum_{P \in B_{1}(q-k)} \int_{P} B_{1, q-k}(x) \chi_{\frac{1}{2} Q}(x) \mathcal{A}_{2^{q-3}}\left(\widetilde{\mathcal{A}}_{Q} g_{2} \cdot h_{Q}\right)(x) d x\right| \\
\leq & \sum_{k=4}^{\infty} \sum_{Q \in \mathcal{D}_{0}} \sum_{P \in B_{1}(q-k)} \frac{1}{|P|} \\
& \times\left|\int_{P} \int_{P} B_{1, q-k}(x) \chi_{\frac{1}{2} Q}(x)\left[\mathcal{A}_{2^{q-3}}\left(\widetilde{\mathcal{A}}_{Q} g_{2} \cdot h_{Q}\right)(x)-\mathcal{A}_{2^{q-3}}\left(\widetilde{\mathcal{A}}_{Q} g_{2} \cdot h_{Q}\right)\left(x^{\prime}\right)\right] d x d x^{\prime}\right| .
\end{aligned}
$$

Observe that in the last line we have used the fact that if $P \in B_{1}(q-k), k \geq 4$ with $P \cap \frac{1}{2} Q \neq \emptyset$, then $P \subset \frac{1}{2} Q$. Therefore $\int_{P} B_{1, q-k} \chi_{\frac{1}{2} Q}(x) d x=0$. Now, write $x^{\prime}=x-y$, then $y \in P-P$. Applying this change of variable we get

$$
\begin{aligned}
I I \leq & \sum_{k=4}^{\infty} \sum_{Q \in \mathcal{D}_{0}} \sum_{P \in B_{1}(q-k)} \frac{1}{|P|} \\
& \times\left|\int_{P-P} \int_{P} B_{1, q-k} \chi_{\frac{1}{2} Q}(x)\left[\mathcal{A}_{2^{q-3}}-\tau_{y} \mathcal{A}_{2^{q-3}}\right]\left(\widetilde{\mathcal{A}}_{Q} g_{2} \cdot h_{Q}\right)(x) d x d y\right| \\
& \sum_{k=4}^{\infty} \sum_{Q \in \mathcal{D}_{0}} \sum_{P \in B_{1}(q-k)} \frac{1}{\left|P_{0}\right|} \\
& \times \int_{P_{0}}\left|\int_{P} B_{1, q-k} \chi_{\frac{1}{2} Q}(x)\left[\mathcal{A}_{2^{q-3}}-\tau_{y} \mathcal{A}_{2^{q-3}}\right]\left(\widetilde{\mathcal{A}}_{Q} g_{2} \cdot h_{Q}\right)(x) d x\right| d y
\end{aligned}
$$

\footnotetext{
${ }^{2}$ Note that we are applying the Calderón-Zygmund decomposition for the functions $f_{1}, f_{2}$. Therefore, $\int_{P} B_{1, q-k}(x) d x=0$ may not imply $\int_{P} B_{1, q-k}(x) \chi_{\frac{1}{2} Q}(x) d x=0$, unless $P \subseteq \frac{1}{2} Q$.
} 
where $P-P \subset P_{0}$ and $l_{P_{0}}=2 l_{P}$. Observe that the quantity within the modulus sign in (23) is a function of $y$. We apply duality for the $L^{1}\left(P_{0}\right)$-norm. For $H \in L^{\infty}\left(P_{0}\right)$ we write $(23)$ as

$$
\begin{aligned}
& \sum_{P \in B_{1}(q-k)} \frac{1}{\left|P_{0}\right|} \int_{P_{0}} \int_{P} B_{1, q-k} \chi_{\frac{1}{2} Q}(x)\left[\mathcal{A}_{2^{q-3}}-\tau_{y} \mathcal{A}_{2^{q-3}}\right]\left(\widetilde{\mathcal{A}}_{Q} g_{2} \cdot h_{Q}\right)(x) d x H(y) d y \\
= & \frac{1}{\left|P_{0}\right|} \int_{P_{0}} \int_{\mathbb{P}_{k}} B_{1, q-k} \chi_{\frac{1}{2} Q}(x)\left[\mathcal{A}_{2^{q-3}}-\tau_{y} \mathcal{A}_{2^{q-3}}\right]\left(\widetilde{\mathcal{A}}_{Q} g_{2} \cdot h_{Q}\right)(x) d x H(y) d y \\
\leq & \|H\|_{L^{\infty}} \frac{1}{\left|P_{0}\right|} \int_{P_{0}}\left|\int_{\mathbb{P}_{k}} B_{1, q-k} \chi_{\frac{1}{2} Q}(x)\left[\mathcal{A}_{2^{q-3}}-\tau_{y} \mathcal{A}_{2^{q-3}}\right]\left(\widetilde{\mathcal{A}}_{Q} g_{2} \cdot h_{Q}\right)(x) d x\right| d y \\
= & \|H\|_{L^{\infty}} \frac{1}{\left|P_{0}\right|} \int_{P_{0}}\left|\int_{\mathbb{R}^{n}} B_{1, q-k} \chi_{\frac{1}{2} Q \cap \mathbb{P}_{k}}(x)\left[\mathcal{A}_{2^{q-3}}-\tau_{y} \mathcal{A}_{2^{q-3}}\right]\left(\widetilde{\mathcal{A}}_{Q} g_{2} \cdot h_{Q}\right)(x) d x\right| d y \\
= & \|H\|_{L^{\infty}} \frac{1}{\left|P_{0}\right|} \int_{P_{0}}\left|\int_{\mathbb{R}^{n}}\left[\mathcal{A}_{2^{q-3}}-\mathcal{A}_{2^{q-3}} \tau_{-y}\right] B_{1, q-k} \chi_{\frac{1}{2} Q \cap \mathbb{P}_{k}}(x)\left(\widetilde{\mathcal{A}}_{Q} g_{2} \cdot h_{Q}\right)(x) d x\right| d y .
\end{aligned}
$$

Observe that for all $k \geq 4$, the side length $l_{P} \leq 2^{q-4}$ and hence we have $|y| \leq 2^{q-3}$. Now, applying [20, Lemma 2.3] we get

$$
\begin{aligned}
I I & \lesssim \sum_{k=4}^{\infty} \sum_{Q \in \mathcal{D}_{0}} \frac{1}{\left|P_{0}\right|} \int_{P_{0}}\left(\frac{|y|}{l_{Q}}\right)^{\eta}|Q|\left\langle B_{1, q-k} \chi_{\mathbb{P}_{k}}\right\rangle_{Q, r_{1}}\left\langle\widetilde{\mathcal{A}}_{Q} g_{2} \cdot h_{Q}\right\rangle_{Q, s_{1}} d y \\
& \leq \sum_{k=4}^{\infty} \sum_{Q \in \mathcal{D}_{0}} \frac{1}{\left|P_{0}\right|} \int_{P_{0}}\left(\frac{|y|}{l_{Q}}\right)^{\eta}|Q|\left\langle B_{1, q-k}\right\rangle_{Q, r_{1}}\left\langle\widetilde{\mathcal{A}}_{Q} g_{2} \cdot h_{Q}\right\rangle_{Q, s_{1}} d y .
\end{aligned}
$$

Further, since $y \in P_{0}$ we have $|y| \lesssim 2^{q-k+1}$. This implies that

$$
B G \lesssim \sum_{k=1}^{\infty} 2^{-\eta k} \sum_{Q \in \mathcal{D}_{0}}|Q|\left\langle B_{1, q-k}\right\rangle_{Q, r_{1}}\left\langle\widetilde{\mathcal{A}}_{Q} g_{2} \cdot h_{Q}\right\rangle_{Q, s_{1}} .
$$

Further, note that

$$
\begin{aligned}
\left\langle\widetilde{\mathcal{A}}_{Q} g_{2} \cdot h_{Q}\right\rangle_{Q, s_{1}}=\left(\frac{1}{|Q|} \int_{Q}\left(\widetilde{\mathcal{A}}_{Q} g_{2}\right)^{s_{1}}(x) h_{Q}^{s_{1}}(x) d x\right)^{\frac{1}{s_{1}}} & \leq\left\|\widetilde{\mathcal{A}}_{Q} g_{2}\right\|_{L^{\infty}}\left\langle h_{Q}\right\rangle_{Q, s_{1}} \\
& \lesssim\left\langle f_{2}\right\rangle_{Q_{0}, r_{2}}\left\langle h_{Q}\right\rangle_{Q, s_{1}}
\end{aligned}
$$

and $\left\langle B_{1, q-k}\right\rangle_{Q, r_{1}} \lesssim\left\langle\chi_{F_{1, q, k}}\right\rangle_{Q, r_{1}}+\left\langle f_{1}\right\rangle_{Q_{0}, r_{1}}\left\langle\chi_{E_{1, q, k}}\right\rangle_{Q, r_{1}}$, where $F_{1, q, k}$ are disjoint subsets of $F_{1}$ and $E_{1, q, k}$ are disjoint subsets of $Q_{0}$. Putting these estimates together we get

$$
\begin{aligned}
B G \lesssim & \left\langle f_{2}\right\rangle_{Q_{0}, r_{2}} \sum_{k=1}^{\infty} 2^{-\eta k} \sum_{Q \in \mathcal{D}_{0}}|Q|\left\langle\chi_{F_{1, q, k}}\right\rangle_{Q, r_{1}}\left\langle h_{Q}\right\rangle_{Q, s_{1}} \\
& +\left\langle f_{1}\right\rangle_{Q_{0}, r_{1}}\left\langle f_{2}\right\rangle_{Q_{0}, r_{2}} \sum_{k=1}^{\infty} 2^{-\eta k} \sum_{Q \in \mathcal{D}_{0}}|Q|\left\langle\chi_{E_{1, q, k}}\right\rangle_{Q, r_{1}}\left\langle h_{Q}\right\rangle_{Q, s_{1}}=: B G_{1}+B G_{2} .
\end{aligned}
$$

We estimate both the terms separately. For the term $B G_{1}$, note that $\left(\frac{1}{r_{1}}, \frac{1}{s_{1}}\right)$ in the interior of $L_{n}$, which implies that $\frac{1}{r_{1}}+\frac{1}{s_{1}}>1$. Choose $\tau>0$ such that $\frac{1}{r_{1}}-\tau+\frac{1}{s_{1}}=1$. Write $\frac{1}{r_{1}}-\tau=\frac{1}{\dot{r}_{1}}$ and note that $\dot{r}_{1}>r_{1}$. We have, by using that $F_{1, q, k} \subseteq F_{1}$ and the stopping time condition for the function $f_{1}$,

$$
\begin{aligned}
\left\langle\chi_{F_{1, q, k}}\right\rangle_{Q, r_{1}}=\left(\frac{1}{|Q|} \int_{Q} \chi_{F_{1, q, k}}^{r_{1}}\right)^{\frac{1}{r_{1}}}\left(\frac{1}{|Q|} \int_{Q} \chi_{F_{1, q, k}}^{r_{1}}\right)^{\tau} & \leq\left(\frac{1}{|Q|} \int_{Q} \chi_{F_{1, q, k}}^{r_{1}}\right)^{\frac{1}{r_{1}}}\left(\frac{1}{|Q|} \int_{Q} f_{1}^{r_{1}}\right)^{\tau} \\
& \lesssim\left(\frac{1}{|Q|} \int_{Q} \chi_{F_{1, q, k}}^{r_{1}}\right)^{\frac{1}{r_{1}}}\left\langle f_{1}\right\rangle_{Q_{0}, r_{1}}^{\tau r_{1}} .
\end{aligned}
$$

Therefore, as $t \geq s_{1}$,

$$
B G_{1}=\left\langle f_{2}\right\rangle_{Q_{0}, r_{2}} \sum_{k=1}^{\infty} 2^{-\eta k} \sum_{Q \in \mathcal{D}_{0}}|Q|\left\langle\chi_{F_{1, q, k}}\right\rangle_{Q, r_{1}}\left\langle h_{Q}\right\rangle_{Q, s_{1}}
$$




$$
\begin{aligned}
& \lesssim\left\langle f_{1}\right\rangle_{Q_{0}, r_{1}}^{\tau r_{1}}\left\langle f_{2}\right\rangle_{Q_{0}, r_{2}} \sum_{k=1}^{\infty} 2^{-\eta k} \sum_{Q \in \mathcal{D}_{0}}|Q|\left(\frac{1}{|Q|} \int_{Q} \chi_{F_{1, q, k}}\right)^{\frac{1}{\dot{r}_{1}}}\left(\frac{1}{|Q|} \int_{Q} h_{Q}^{s_{1}}\right)^{\frac{1}{s_{1}}} \\
& \lesssim\left\langle f_{1}\right\rangle_{Q_{0}, r_{1}}^{\tau r_{1}}\left\langle f_{2}\right\rangle_{Q_{0}, r_{2}} \sum_{k=1}^{\infty} 2^{-\eta k}\left(\sum_{Q_{0} \mathcal{D}_{0}} \int_{Q} \chi_{F_{1, q, k}}\right)^{\frac{1}{\dot{r}_{1}}}\left(\sum_{Q_{Q} \in \mathcal{D}_{0}} \int_{Q} h^{s_{1}} \chi_{B_{Q}}\right)^{\frac{1}{s_{1}}} \\
& \lesssim\left\langle f_{1}\right\rangle_{Q_{0}, r_{1}}^{\tau r_{1}}\left\langle f_{2}\right\rangle_{Q_{0}, r_{2}}\left\langle f_{1}\right\rangle_{Q_{0}, r_{1}}^{\frac{r_{1}}{\dot{r}_{1}}}\left|Q_{0}\right|^{\frac{1}{\dot{r}_{1}}}\langle h\rangle_{Q_{0}, s_{1}}\left|Q_{0}\right|^{\frac{1}{s_{1}}} \\
& \leq\left\langle f_{1}\right\rangle_{Q_{0}, r_{1}}\left\langle f_{2}\right\rangle_{Q_{0}, r_{2}}\langle h\rangle_{Q_{0}, t}\left|Q_{0}\right| .
\end{aligned}
$$

Next, the term $B G_{2}$ may be estimated as follows. Since $\dot{r}_{1}>r_{1}$, we have $\left\langle\chi_{E_{1, q, k}}\right\rangle_{Q, r_{1}} \leq$ $\left\langle\chi_{E_{1, q, k}}\right\rangle_{Q, \dot{r}_{1}}$. Consider

$$
\begin{aligned}
B G_{2} & =\left\langle f_{1}\right\rangle_{Q_{0}, r_{1}}\left\langle f_{2}\right\rangle_{Q_{0}, r_{2}} \sum_{k=1}^{\infty} 2^{-\eta k} \sum_{Q \in \mathcal{D}_{0}}|Q|\left\langle\chi_{E_{1, q, k}}\right\rangle_{Q, r_{1}}\left\langle h_{Q}\right\rangle_{Q, s_{1}} \\
& \leq\left\langle f_{1}\right\rangle_{Q_{0}, r_{1}}\left\langle f_{2}\right\rangle_{Q_{0}, r_{2}} \sum_{k=1}^{\infty} 2^{-\eta k} \sum_{Q \in \mathcal{D}_{0}}|Q|\left\langle\chi_{E_{1, q, k}}\right\rangle_{Q, \dot{r}_{1}}\left\langle h_{Q}\right\rangle_{Q, s_{1}} \\
& \leq\left\langle f_{1}\right\rangle_{Q_{0}, r_{1}}\left\langle f_{2}\right\rangle_{Q_{0}, r_{2}} \sum_{k=1}^{\infty} 2^{-\eta k}\left(\sum_{Q \in \mathcal{D}_{0}} \int_{Q} \chi_{E_{1, q, k}}\right)^{\frac{1}{\dot{r}_{1}}}\left(\sum_{Q \in \mathcal{D}_{0}} \int_{Q} h^{s_{1}} \chi_{B_{Q}}\right)^{\frac{1}{s_{1}}} \\
& \lesssim\left\langle f_{1}\right\rangle_{Q_{0}, r_{1}}\left\langle f_{2}\right\rangle_{Q_{0}, r_{2}}\left|Q_{0}\right|^{\frac{1}{\dot{r}_{1}}}\langle h\rangle_{Q_{0}, s_{1}}\left|Q_{0}\right|^{\frac{1}{s_{1}}} \\
& \leq\left\langle f_{1}\right\rangle_{Q_{0}, r_{1}}\left\langle f_{2}\right\rangle_{Q_{0}, r_{2}}\langle h\rangle_{Q_{0}, t}\left|Q_{0}\right| \text { as } t \geq s_{1} .
\end{aligned}
$$

Estimates (24) and (25) yield the desired result for the term $B G$. The estimate for the third term $G B$ follows similarly.

Estimate for $B B$ (both functions are bad) part: We have

$$
\begin{aligned}
B B & =\left|\sum_{Q \in \mathcal{D}_{0}}\left\langle\widetilde{\mathcal{A}}_{Q} b_{1} \widetilde{\mathcal{A}}_{Q} b_{2}, h_{Q}\right\rangle\right|=\left|\sum_{Q \in \mathcal{D}_{0}} \int b_{1}(x)\left(\widetilde{\mathcal{A}}_{Q}\right)^{*}\left(\widetilde{\mathcal{A}}_{Q} b_{2} \cdot h_{Q}\right)(x) d x\right| \\
& =\left|\sum_{Q \in \mathcal{D}_{0}} \sum_{k=1}^{\infty} \sum_{P \in B_{1}(q-k)} \int_{P} B_{1, q-k}(x) \chi_{\frac{1}{2} Q}(x) \mathcal{A}_{2^{q-3}}\left(\widetilde{\mathcal{A}}_{Q} b_{2} \cdot h_{Q}\right)(x) d x\right| \\
& \leq\left|\sum_{Q \in \mathcal{D}_{0}} \sum_{k=1}^{3} \sum_{P \in B_{1}(q-k)} \int_{P} B_{1, q-k}(x) \chi_{\frac{1}{2} Q}(x) \mathcal{A}_{2^{q-3}}\left(\widetilde{\mathcal{A}}_{Q} b_{2} \cdot h_{Q}\right)(x) d x\right| \\
& +\left|\sum_{Q \in \mathcal{D}_{0}} \sum_{k=4}^{\infty} \sum_{P \in B_{1}(q-k)} \int_{P} B_{1, q-k}(x) \chi_{\frac{1}{2} Q}(x) \mathcal{A}_{2^{q-3}}\left(\widetilde{\mathcal{A}}_{Q} b_{2} \cdot h_{Q}\right)(x) d x\right| \\
& =I_{b b}+I I_{b b} .
\end{aligned}
$$

Now $I_{b b}$ can be handled similarly as $I$. On the other hand, we estimate $I I_{b b}$ as follows

$$
\begin{aligned}
I I_{b b} \leq & \sum_{k=4}^{\infty} \sum_{Q \in \mathcal{D}_{0}} \sum_{P \in B_{1}(q-k)} \frac{1}{|P|} \\
& \times\left|\int_{P} \int_{P} B_{1, q-k} \chi_{\frac{1}{2} Q}(x)\left[\mathcal{A}_{2^{q-3}}\left(\widetilde{\mathcal{A}}_{Q} b_{2} \cdot h_{Q}\right)(x)-\mathcal{A}_{2^{q-3}}\left(\widetilde{\mathcal{A}}_{Q} b_{2} \cdot h_{Q}\right)\left(x^{\prime}\right)\right] d x d x^{\prime}\right| \\
& \lesssim \sum_{k=4}^{\infty} \sum_{Q \in \mathcal{D}_{0}} \sum_{P \in B_{1}(q-k)} \frac{1}{|P|} \\
& \times\left|\int_{P-P} \int_{P} B_{1, q-k} \chi_{\frac{1}{2} Q}(x)\left[\mathcal{A}_{2^{q-3}}-\tau_{y} \mathcal{A}_{2^{q-3}}\right]\left(\widetilde{\mathcal{A}}_{Q} b_{2} \cdot h_{Q}\right)(x) d x d y\right| .
\end{aligned}
$$

Now, we proceed as for $B G$, but at the last line apply Hölder's inequality. Then the term is dominated by 


$$
\begin{aligned}
\sum_{k=4}^{\infty} \sum_{Q \in \mathcal{D}_{0}} \frac{1}{\left|P_{0}\right|} \int_{P_{0}} \|\left[\mathcal{A}_{2^{q-3}}\right. & \left.-\mathcal{A}_{2^{q-3}} \tau_{-y}\right] B_{1, q-k} \chi_{\mathbb{P}_{k}}\left\|_{L^{s_{1}}}\right\| \widetilde{\mathcal{A}}_{Q} b_{2}\left\|_{L^{s_{2}^{\prime}}}\right\| h_{Q} \|_{L^{t}} \\
& \lesssim \sum_{k=4}^{\infty} \sum_{Q \in \mathcal{D}_{0}} \frac{1}{\left|P_{0}\right|} \int_{P_{0}}\left(\frac{|y|}{l_{Q}}\right)^{\eta}|Q|^{1-\frac{1}{s_{1}}}\left\langle B_{1, q-k}\right\rangle_{Q, r_{1}}\left\|\widetilde{\mathcal{A}}_{Q} b_{2}\right\|_{L^{s_{2}^{\prime}}}\left\|h_{Q}\right\|_{L^{t}}
\end{aligned}
$$

where, in the last inequality, we have used [20, Theorem 2.1] for $\left(\frac{1}{r_{1}}, \frac{1}{s_{1}^{\prime}}\right)$ in the interior of $L_{n}^{\prime}=\left\{\left(\frac{1}{r}, \frac{1}{s}\right):\left(\frac{1}{r}, 1-\frac{1}{s}\right) \in L_{n}\right\}$ and Hölder's inequality with exponents $\frac{1}{t^{\prime}}=\frac{1}{s_{1}^{\prime}}+\frac{1}{s_{2}^{\prime}}$. This yields

$$
B B \leq \sum_{k=1}^{\infty} 2^{-\eta k} \sum_{Q \in \mathcal{D}_{0}}|Q|^{1-\frac{1}{s_{1}}}\left\langle B_{1, q-k}\right\rangle_{Q, r_{1}}\left\|\widetilde{\mathcal{A}}_{Q} b_{2}\right\|_{L^{s_{2}^{\prime}}}\left\|h_{Q}\right\|_{L^{t}}
$$

Next, we make use of [20, Lemma 2.3] to estimate the quantity

$$
\left\|\widetilde{\mathcal{A}}_{Q} b_{2}\right\|_{L^{s_{2}^{\prime}}}=\sup _{\|\psi\|_{L^{s_{2}}=1}}\left|\left\langle\widetilde{\mathcal{A}}_{Q} b_{2}, \psi\right\rangle\right| .
$$

Indeed, with a similar computation as the one for $B G$, we can conclude

$$
\begin{aligned}
\left|\left\langle\widetilde{\mathcal{A}}_{Q} b_{2}, \psi\right\rangle\right| & =\left|\int \widetilde{\mathcal{A}}_{Q} b_{2}(x) \psi(x) d x\right|=\left|\sum_{j=1}^{\infty} \sum_{P \in B_{2}(q-j)} \int_{P} B_{2, q-j}(x)\left(\widetilde{\mathcal{A}}_{Q}\right)^{*} \psi(x) d x\right| \\
& =\left|\sum_{j=1}^{\infty} \sum_{P \in B_{2}(q-j)} \int_{P} B_{2, q-j}(x) \chi_{\frac{1}{2} Q}(x) \mathcal{A}_{2^{q-3}} \psi(x) d x\right| \\
& \lesssim \sum_{j=1}^{3}|Q|^{1-\frac{1}{s_{2}}}\left\langle B_{2, q-j}\right\rangle_{Q, r_{2}}|| \psi\left\|_{L^{s_{2}}}+\sum_{j=4}^{\infty} \frac{1}{\left|P_{0}\right|} \int_{P_{0}}|Q|^{1-\frac{1}{s_{2}}}\left(\frac{|y|}{l_{Q}}\right)^{\eta}\left\langle B_{2, q-j}\right\rangle_{Q, r_{2}}\right\| \psi \|_{L^{s_{2}}} d y .
\end{aligned}
$$

Thus we obtain the following estimate.

$$
B B \lesssim \sum_{k, j=1}^{\infty} 2^{-\eta(k+j)} \sum_{Q \in \mathcal{D}_{0}} \frac{|Q|^{2}}{|Q|^{\frac{1}{s_{1}}+\frac{1}{s_{2}}}}\left\langle B_{1, q-k}\right\rangle_{Q, r_{1}}\left\langle B_{2, q-j}\right\rangle_{Q, r_{2}}\left(\int_{Q} h_{Q}^{t}\right)^{\frac{1}{t}}
$$

where we know that

$$
\left\langle B_{1, q-k}\right\rangle_{Q, r_{1}} \lesssim\left\langle\chi_{F_{1, q, k}}\right\rangle_{Q, r_{1}}+\left\langle f_{1}\right\rangle_{Q_{0}, r_{1}}\left\langle\chi_{E_{1, q, k}}\right\rangle_{Q, r_{1}}
$$

and

$$
\left\langle B_{2, q-j}\right\rangle_{Q, r_{2}} \lesssim\left\langle\chi_{F_{2, q, j}}\right\rangle_{Q, r_{2}}+\left\langle f_{2}\right\rangle_{Q_{0}, r_{2}}\left\langle\chi_{E_{2, q, j}}\right\rangle_{Q, r_{2}}
$$

Here, $E_{1, q, k}, E_{2, q, j}$ are disjoint subsets of $Q_{0}$ and $F_{1, q, k}, F_{2, q, j}$ are disjoint subsets of $F_{1}, F_{2}$, respectively.

Substituting (26) and (27) into (6.3), we get the following four terms, which will be estimated separately.

$$
\begin{aligned}
B B \lesssim & \sum_{k, j=1}^{\infty} 2^{-\eta(k+j)} \sum_{Q \in \mathcal{D}_{0}} \frac{|Q|^{2}}{|Q|^{\frac{1}{s_{1}}+\frac{1}{s_{2}}}}\left\langle\chi_{F_{1, q, k}}\right\rangle_{Q, r_{1}}\left\langle\chi_{F_{2, q, j}}\right\rangle_{Q, r_{2}}\left(\int_{Q} h_{Q}^{t}\right)^{\frac{1}{t}} \\
& +\sum_{k, j=1}^{\infty} 2^{-\eta(k+j)} \sum_{Q \in \mathcal{D}_{0}} \frac{|Q|^{2}}{|Q|^{\frac{1}{s_{1}}+\frac{1}{s_{2}}}}\left\langle\chi_{F_{1, q, k}}\right\rangle_{Q, r_{1}}\left\langle f_{2}\right\rangle_{Q_{0}, r_{2}}\left\langle\chi_{E_{2, q, j}}\right\rangle_{Q, r_{2}}\left(\int_{Q} h_{Q}^{t}\right)^{\frac{1}{t}} \\
& +\sum_{k, j=1}^{\infty} 2^{-\eta(k+j)} \sum_{Q \in \mathcal{D}_{0}} \frac{|Q|^{2}}{|Q|^{\frac{1}{s_{1}}+\frac{1}{s_{2}}}}\left\langle f_{1}\right\rangle_{Q_{0}, r_{1}}\left\langle\chi_{E_{1, q, k}}\right\rangle_{Q, r_{1}}\left\langle\chi_{F_{2, q, j}}\right\rangle_{Q, r_{2}}\left(\int_{Q} h_{Q}^{t}\right)^{\frac{1}{t}} \\
& +\sum_{k, j=1}^{\infty} 2^{-\eta(k+j)} \sum_{Q \in \mathcal{D}_{0}} \frac{|Q|^{2}}{|Q|^{\frac{1}{s_{1}}+\frac{1}{s_{2}}}}\left\langle f_{1}\right\rangle_{Q_{0}, r_{1}}\left\langle\chi_{E_{1, q, k}}\right\rangle_{Q, r_{1}}\left\langle f_{2}\right\rangle_{Q_{0}, r_{2}}\left\langle\chi_{E_{2, q, j}}\right\rangle_{Q, r_{2}}\left(\int_{Q} h_{Q}^{t}\right)^{\frac{1}{t}} \\
= & : B B_{1}+B B_{2}+B B_{3}+B B_{4} .
\end{aligned}
$$


Estimate for the first term $B B_{1}$. At this point, one has to deal with the cases $\frac{1}{r_{1}}+\frac{1}{r_{2}}>1$ and $\frac{1}{r_{1}}+\frac{1}{r_{2}} \leq 1$ separately. Let us start with the case $\frac{1}{r_{1}}+\frac{1}{r_{2}}>1$. Choose positive numbers $\tau_{1}$ and $\tau_{2}$ such that $\frac{1}{r_{1}}+\frac{1}{r_{2}}=1+\tau_{1}+\tau_{2}$ and denote $\frac{1}{r_{i}}-\tau_{i}=\frac{1}{\dot{r}_{i}}, i=1,2$. Note that $\frac{1}{\dot{r}_{1}}+\frac{1}{\dot{r}_{2}}=1$. We have

$$
\begin{aligned}
B B_{1} & \lesssim\langle h\rangle_{Q_{0}, t} \sum_{k, j=1}^{\infty} 2^{-\eta(k+j)} \sum_{Q \in \mathcal{D}_{0}}|Q|^{1-\frac{1}{r_{1}}-\frac{1}{r_{2}}}\left(\int_{Q} \chi_{F_{1, q, k}}\right)^{\frac{1}{r_{1}}}\left(\int_{Q} \chi_{F_{2, q, j}}\right)^{\frac{1}{r_{2}}} \\
& \lesssim\langle h\rangle_{Q_{0}, t}\left\langle f_{1}\right\rangle_{Q_{0}, r_{1}}^{\tau_{1} r_{1}}\left\langle f_{2}\right\rangle_{Q_{0}, r_{2}}^{\tau_{2} r_{2}}\left\langle f_{1}\right\rangle_{Q_{0}, r_{1}}^{\frac{r_{1}}{\dot{r}_{1}}}\left\langle f_{2}\right\rangle_{Q_{0}, r_{2}}^{\frac{r_{2}}{r_{2}}}\left|Q_{0}\right|=\left\langle f_{1}\right\rangle_{Q_{0}, r_{1}}\left\langle f_{2}\right\rangle_{Q_{0}, r_{2}}\langle h\rangle_{Q_{0}, t}\left|Q_{0}\right| .
\end{aligned}
$$

The case $\frac{1}{r_{1}}+\frac{1}{r_{2}}=1$ follows similarly with $\tau_{1}=\tau_{2}=0$.

Let us turn to the case when $\frac{1}{r_{1}}+\frac{1}{r_{2}}<1$. Observe that $\frac{1}{r_{1}}+\frac{1}{r_{2}}+\frac{1}{t}>1$. Now, choose $\tau_{1}, \tau_{2}>0$ such that $\frac{1}{r_{1}}+\frac{1}{r_{2}}+\frac{1}{t}=1+\tau_{1}+\tau_{2}$. This implies $\frac{1}{\dot{r}_{1}}+\frac{1}{\dot{r}_{2}}+\frac{1}{t}=1$, where $\frac{1}{\dot{r}_{i}}=\frac{1}{r_{i}}-\tau_{i}$, for $i=1,2$.

$$
\begin{aligned}
& B B_{1}=\sum_{k, j=1}^{\infty} 2^{-\eta(k+j)} \sum_{Q \in \mathcal{D}_{0}}|Q|^{1-\frac{1}{t}}\left\langle\chi_{F_{1, q, k}}\right\rangle_{Q, r_{1}}\left\langle\chi_{F_{2, q, j}}\right\rangle_{Q, r_{2}}\left(\int_{Q} h_{Q}^{t}\right)^{\frac{1}{t}} \\
& \lesssim\left\langle f_{1}\right\rangle_{Q_{0}, r_{1}}^{\tau_{1} r_{1}}\left\langle f_{2}\right\rangle_{Q_{0}, r_{2}}^{\tau_{2} r_{2}} \sum_{k, j=1}^{\infty} 2^{-\eta(k+j)} \sum_{Q \in \mathcal{D}_{0}}\left(\int_{Q} \chi_{F_{1, q, k}}\right)^{\frac{1}{\dot{r}_{1}}}\left(\int_{Q} \chi_{F_{2, q, j}}\right)^{\frac{1}{\dot{r}_{2}}}\left(\int_{Q} h_{Q}^{t}\right)^{\frac{1}{t}} \\
& \lesssim\left\langle f_{1}\right\rangle_{Q_{0}, r_{1}}\left\langle f_{2}\right\rangle_{Q_{0}, r_{2}}\langle h\rangle_{Q_{0}, t}\left|Q_{0}\right| .
\end{aligned}
$$

In the last inequality we have used the Hölder's inequality with respect to $\dot{r_{1}}, \dot{r_{2}}$ and $t$.

The latter case is analogous for the remaining three terms $B B_{2}, B B_{3}$ and $B B_{4}$, hence we will focus on the estimates only for the case when $\frac{1}{r_{1}}+\frac{1}{r_{2}}>1$.

Estimate for the second and third terms $B B_{2}$ and $B B_{3}$. The estimates for $B B_{2}$ and $B B_{3}$ may be obtained in a similar fashion. We provide here the argument for the term $B B_{3}$.

Since $\frac{1}{r_{1}}+\frac{1}{r_{2}}>1$, we can choose a positive number $\tau$ such that $\frac{1}{r_{1}}-\tau+\frac{1}{r_{2}}=1$. Denote $\frac{1}{r_{1}}-\tau=\frac{1}{\dot{r}_{1}}$ and note that $\frac{1}{\dot{r}_{1}}+\frac{1}{r_{2}}=1$ and $r_{1}<\dot{r}_{1}$. Then we have,

$$
\begin{aligned}
B B_{3} & \lesssim\left\langle f_{1}\right\rangle_{Q_{0}, r_{1}}\langle h\rangle_{Q_{0}, t} \sum_{k, j=1}^{\infty} 2^{-\eta(k+j)} \sum_{Q \in \mathcal{D}_{0}}|Q|^{1-\frac{1}{r_{1}}-\frac{1}{r_{2}}+\tau}\left(\int_{Q} \chi_{E_{1, q, k}}\right)^{\frac{1}{\dot{r}_{1}}}\left(\int_{Q} \chi_{F_{2, q, j}}\right)^{\frac{1}{r_{2}}} \\
& \leq\left\langle f_{1}\right\rangle_{Q_{0}, r_{1}}\langle h\rangle_{Q_{0}, t} \sum_{k, j=1}^{\infty} 2^{-\eta(k+j)}\left(\sum_{Q \in \mathcal{D}_{0}} \int_{Q} \chi_{E_{1, q, k}}\right)^{\frac{1}{\dot{r}_{1}}}\left(\sum_{Q \in \mathcal{D}_{0}} \int_{Q} \chi_{F_{2, q, j}}\right)^{\frac{1}{r_{2}}} \\
& \lesssim\left\langle f_{1}\right\rangle_{Q_{0}, r_{1}}\langle h\rangle_{Q_{0}, t}\left|Q_{0}\right|^{\frac{1}{\dot{r}_{1}}}\left\langle f_{2}\right\rangle_{Q_{0}, r_{2}}\left|Q_{0}\right|^{\frac{1}{r_{2}}}=\left\langle f_{1}\right\rangle_{Q_{0}, r_{1}}\left\langle f_{2}\right\rangle_{Q_{0}, r_{2}}\langle h\rangle_{Q_{0}, t}\left|Q_{0}\right| .
\end{aligned}
$$

Estimate for the fourth term $B B_{4}$. Choose $\tau_{1}$ and $\tau_{2}$ as in the case $B B_{1}$. Consider

$$
\begin{aligned}
B B_{4} & \lesssim\left\langle f_{1}\right\rangle_{Q_{0}, r_{1}}\left\langle f_{2}\right\rangle_{Q_{0}, r_{2}}\langle h\rangle_{Q_{0}, t} \sum_{k, j=1}^{\infty} 2^{-\eta(k+j)} \sum_{Q \in \mathcal{D}_{0}}|Q|^{1-\frac{1}{r_{1}}-\frac{1}{r_{2}}}\left(\int_{Q} \chi_{E_{1, q, k}}\right)^{\frac{1}{r_{1}}}\left(\int_{Q} \chi_{E_{2, q, j}}\right)^{\frac{1}{r_{2}}} \\
& \leq\left\langle f_{1}\right\rangle_{Q_{0}, r_{1}}\left\langle f_{2}\right\rangle_{Q_{0}, r_{2}}\langle h\rangle_{Q_{0}, t} \sum_{k, j=1}^{\infty} 2^{-\eta(k+j)}\left(\sum_{Q \in \mathcal{D}_{0}} \int_{Q} \chi_{E_{1, q, k}}\right)^{\frac{1}{\dot{r}_{1}}}\left(\sum_{Q \in \mathcal{D}_{0}} \int_{Q} \chi_{E_{2, q, j}}\right)^{\frac{1}{\dot{r}_{2}}} \\
& \lesssim\left\langle f_{1}\right\rangle_{Q_{0}, r_{1}}\left\langle f_{2}\right\rangle_{Q_{0}, r_{2}}\langle h\rangle_{Q_{0}, t}\left|Q_{0}\right| .
\end{aligned}
$$

This completes the proof of Lemma 6.2 for the lacunary bilinear spherical maximal operator.

For the case of the full bilinear spherical maximal operator, we proceed analogously as in the proof of the lacunary maximal operator. In this case, we have to deal with

$$
\widetilde{\widetilde{\mathcal{M}}}_{Q}\left(f_{1}, f_{2}\right)(x):=\sup _{2^{q-4} \leq t \leq 2^{q-3}} \mathcal{A}_{t}\left(f_{1} \chi_{\frac{1}{2} Q}\right)(x) \mathcal{A}_{t}\left(f_{2} \chi_{\frac{1}{2} Q}\right)(x)
$$

and we call

$$
\widetilde{\mathcal{A}}_{t} f_{i}(x)=\mathcal{A}_{t}\left(f_{i} \chi_{\frac{1}{2} Q}\right)(x)
$$


We use the Calderón-Zygmund decomposition to write $f_{i}=g_{i}+b_{i}, \quad i=1,2$ to get the following

$$
\begin{aligned}
\left|\sum_{Q \in \mathcal{D}_{0}}\left\langle\widetilde{\widetilde{\mathcal{M}}}_{Q}\left(f_{1}, f_{2}\right), h_{Q}\right\rangle\right| \leq\left|\sum_{Q \in \mathcal{D}_{0}}\left\langle\widetilde{\widetilde{\mathcal{M}}}_{Q}\left(g_{1}, g_{2}\right), h_{Q}\right\rangle\right|+\left|\sum_{Q \in \mathcal{D}_{0}}\left\langle\widetilde{\widetilde{\mathcal{M}}}_{Q}\left(g_{1}, b_{2}\right), h_{Q}\right\rangle\right| \\
+\left|\sum_{Q \in \mathcal{D}_{0}}\left\langle\widetilde{\widetilde{\mathcal{M}}}_{Q}\left(b_{1}, g_{2}\right), h_{Q}\right\rangle\right|+\left|\sum_{Q \in \mathcal{D}_{0}}\left\langle\widetilde{\widetilde{\mathcal{M}}}_{Q}\left(b_{1}, b_{2}\right), h_{Q}\right\rangle\right| \\
=: G G+G B+B G+B B .
\end{aligned}
$$

Estimate for $G G$ (both functions good). In this case we have

$$
\begin{aligned}
\sum_{Q \in \mathcal{D}_{0}}\left|\left\langle\widetilde{\widetilde{\mathcal{M}}}_{Q}\left(g_{1}, g_{2}\right), h_{Q}\right\rangle\right| & \leq \sum_{Q \in \mathcal{D}_{0}}\left\|\widetilde{\widetilde{M}}_{Q} g_{1}\right\|_{L^{\infty}}\left\|\widetilde{\widetilde{M}}_{Q} g_{2}\right\|_{L^{\infty}}\left\|h_{Q}\right\|_{L^{1}} \\
& \lesssim\left\langle f_{1}\right\rangle_{Q_{0}, r_{1}}\left\langle f_{2}\right\rangle_{Q_{0}, r_{2}} \sum_{Q \in \mathcal{D}_{0}} \int|h(x)| \chi_{B_{Q}}(x) d x \\
& \lesssim\left\langle f_{1}\right\rangle_{Q_{0}, r_{1}}\left\langle f_{2}\right\rangle_{Q_{0}, r_{2}}\langle h\rangle_{Q_{0}}\left|Q_{0}\right| .
\end{aligned}
$$

Estimate for $B G$ (one function good and one function bad). We have, proceeding as in the case of $B G$ for the lacunary spherical maximal function. Let $t_{Q}: Q \rightarrow\left[2^{q-4}, 2^{q-3}\right]$ be a measurable function, then

$$
\begin{aligned}
& \left|\sum_{Q \in \mathcal{D}_{0}}\left\langle\widetilde{\widetilde{\mathcal{M}}}_{Q}\left(b_{1}, g_{2}\right), h_{Q}\right\rangle\right| \leq \sum_{Q \in \mathcal{D}_{0}}\left|\int b_{1}(x)\left(\widetilde{\mathcal{A}}_{t_{Q}}\right)^{*}\left(\widetilde{\mathcal{A}}_{t_{Q}} g_{2} \cdot h_{Q}\right)(x) d x\right| \\
& \lesssim \sum_{k=1}^{3} \sum_{Q \in \mathcal{D}_{0}}|Q|\left\langle B_{1, q-k}\right\rangle_{Q, r_{1}}\left\langle\widetilde{\mathcal{A}}_{t_{Q}} g_{2} \cdot h_{Q}\right\rangle_{Q, s_{1}} \\
& \quad+\sum_{k \geq 4} \sum_{Q \in \mathcal{D}_{0}} \frac{1}{\left|P_{0}\right|} \int_{P_{0}}\left(\frac{|y|}{l_{Q}}\right)^{\eta}|Q|\left\langle B_{1, q-k}\right\rangle_{Q, r_{1}}\left\langle\widetilde{\mathcal{A}}_{t_{Q}} g_{2} \cdot h_{Q}\right\rangle_{Q, s_{1}} d y \\
& \lesssim \sum_{k \geq 1} 2^{-k \eta} \sum_{Q \in \mathcal{D}_{0}}|Q|\left\langle B_{1, q-k}\right\rangle_{Q, r_{1}}\left\langle\widetilde{\mathcal{A}}_{t_{Q}} g_{2} \cdot h_{Q}\right\rangle_{Q, s_{1}},
\end{aligned}
$$

where we have used [20, Theorem 3.2] in the second to last inequality. Next, observe that

$$
\left\langle\widetilde{\mathcal{A}}_{t_{Q}} g_{2} \cdot h_{Q}\right\rangle_{Q, s_{1}} \lesssim\left\langle f_{2}\right\rangle_{Q_{0}, r_{2}}\left\langle h_{Q}\right\rangle_{Q, s_{1}}
$$

This point onward, we can follow the proof in the case of bilinear lacunary spherical maximal function and get the desired estimates. We skip the details.

This completes the proof of Lemma 6.2.

\section{NeCESSARY CONDITIONS FOR THE SPARSE DOMINATION}

In this section we prove the necessity part of Theorem 2.3. Indeed, we discuss the relations involving the exponents $r_{1}, r_{2}, s_{1}, s_{2}$ and $t$ and show that they are necessary conditions for the validity of the sparse domination of the bilinear (both lacunary and full) spherical maximal functions. We make use of examples in the spirit of Knapp and Stein [32]. The approach in the (linear) sparse domination setting is developed in [20].

7.1. Sparse form for $\mathcal{M}_{\text {lac }}$. Let $f_{1}=f_{2}=\chi_{|| x|-1|<\delta}$ and $h=\chi_{|x| \leq c \delta}$ for some $0<\delta<1 / 4$ and $c \in\left(0, \frac{1}{2}\right)$. Then we get that $\mathcal{A}_{1} f_{i}(x) \geq c h(x), i=1,2$. Therefore, the sparse domination for the operator $\mathcal{M}_{\text {lac }}$ implies that

$$
\delta^{n} \lesssim \int_{\mathbb{R}^{n}} \mathcal{A}_{1} f_{1}(x) \mathcal{A}_{1} f_{2}(x) h(x) d x \leq C_{0} \sum_{Q \in \mathcal{S}}|Q|\left\langle f_{1}\right\rangle_{Q, r_{1}}\left\langle f_{2}\right\rangle_{Q, r_{2}}\langle h\rangle_{Q, t}
$$

where $\mathcal{S}$ is a sparse collection. Observe that in the estimate above, in order to make non-trivial contribution to the term on the right hand side, the cube $Q \in \mathcal{S}$ must necessarily intersect with 
the supports of $f_{1}, f_{2}$ and $h$. Moreover, it is crucial that

$$
\operatorname{dist}\left(\operatorname{supp}\left(f_{i}\right), \operatorname{supp}(h)\right) \geq 1 / 2,
$$

which implies that all the cubes contributing to the sum have side length at least $1 / 2$. Further, since the contribution of a cube decreases as its size increases, it suffices to assume that $\mathcal{S}$ consists of one such cube $Q$. We have the estimate

$$
\delta^{n} \lesssim\left\|f_{1}\right\|_{L^{r_{1}}}\left\|f_{2}\right\|_{L^{r_{2}}}\|h\|_{L^{t}} \lesssim|Q|^{1-\frac{1}{r_{1}}-\frac{1}{r_{2}}-\frac{1}{t}} \delta^{\frac{1}{r_{1}}+\frac{1}{r_{2}}+\frac{n}{t}} .
$$

Since $\delta>0$ can be chosen arbitrarily small, we get that

$$
\frac{1}{r_{1}}+\frac{1}{r_{2}}+\frac{n}{t} \leq n
$$

Note that the estimate above forces the condition $t>1$. Substituting the value of $t$ in terms of $s_{1}$ and $s_{2}$, we get the following necessary condition

$$
\frac{1}{r_{1}}+\frac{n}{s_{1}}+\frac{1}{r_{2}}+\frac{n}{s_{2}} \leq 2 n .
$$

In a similar fashion, one can show that if $f_{1}=f_{2}=\chi_{|x|<\delta}$ and $h=\chi_{|| x|-1|<c \delta}$ for some $0<\delta<1 / 4$ and $0<c<\frac{1}{2}$, then we get that $\mathcal{A}_{1} f_{1}(x) \geq c \delta^{n-1} h(x)$. This gives us another necessary condition, namely $\frac{n}{r_{1}}+\frac{n}{r_{2}}+\frac{1}{t} \leq 2 n-1$. This would mean that

$$
\frac{n}{r_{1}}+\frac{1}{s_{1}}+\frac{n}{r_{2}}+\frac{1}{s_{2}} \leq 2 n \text {. }
$$

The conditions (28) and (29) imply that both of $\left(\frac{1}{r_{i}}, \frac{1}{s_{i}}\right), i=1,2$, cannot lie outside of the triangle $L_{n}$.

Next, take $f_{1}=\chi_{|x|<\delta}, f_{2}=\chi_{|x|<2}$ (also interchanging $f_{1}$ and $f_{2}$ ) and $h=\chi_{|| x|-1|<c \delta}$ for some $0<\delta<1$ and $0<c<\frac{1}{2}$ and observe that

$$
\delta^{n-1} \delta \lesssim \delta^{\frac{n}{r_{1}}} \delta^{\frac{1}{t}}
$$

This yields that $\frac{n}{r_{i}}+\frac{1}{t} \leq n, i=1,2$. Similarly, by taking $f_{1}=\chi_{|| x|-1|<\delta}, f_{2}=\chi_{|x|<2}, h=\chi_{|x|<c \delta}$ for some $0<\delta<1 / 4$ and $0<c<\frac{1}{2}$ and interchanging the roles of $f_{1}$ and $f_{2}$ we get that $\frac{1}{r_{i}}+\frac{n}{t} \leq n, i=1,2$.

Putting the above two conditions together we get the following condition.

$$
\max \left\{\frac{n}{r_{i}}+\frac{1}{t}, \frac{1}{r_{i}}+\frac{n}{t}\right\} \leq n, \quad i=1,2 .
$$

The conditions (28), (29) and (30) must necessarily be satisfied for the sparse domination of the operator $\mathcal{M}_{\text {lac }}$ to hold.

7.2. Sparse form for $\mathcal{M}_{\text {full }}$. Consider $f_{1}=|x|^{1-n}\left(\log \frac{1}{|x|}\right)^{-1} \chi_{|x|<\frac{3}{4}}$ and $f_{2}=\chi_{|x|<1}$ and note that $f_{1} \in L^{r_{1}}\left(\mathbb{R}^{n}\right)$ for $1<r_{1} \leq \frac{n}{n-1}$. It is easy to verify that $\mathcal{M}_{\text {full }}\left(f_{1}, f_{2}\right)$ is infinite on a set of positive measure. This gives us the condition that $\frac{1}{r_{1}}<\frac{n-1}{n}$. Using the symmetry between $f_{1}$ and $f_{2}$, we also have that $\frac{1}{r_{2}}<\frac{n-1}{n}$. Next, we observe that both of $\left(\frac{1}{r_{i}}, \frac{1}{s_{i}}\right), i=1,2$ cannot lie above the line segment $P_{1} P_{4}$ in $F_{n}$, see Figure 1 . This can be proved by considering the functions $f_{1}=f_{2}=\chi_{|| x|-1|<\delta}$ and $h=\chi_{|x| \leq c \delta}$ for some $0<\delta<1 / 4$ and $c \in\left(0, \frac{1}{2}\right)$. This is same as in the case of lacunary maximal function. Note that the support-separation property also holds. We omit the details.

Consider $f_{1}=f_{2}=\chi_{R_{1}}$ and $h=\chi_{R_{2}}$, where $R_{1}=[-C \sqrt{\delta}, C \sqrt{\delta}]^{n-1} \times[-C \delta, C \delta]$ and $R_{2}=[-\sqrt{\delta}, \sqrt{\delta}]^{n-1} \times\left[\frac{4}{3}, \frac{5}{3}\right]$. This yields

$$
\left\langle\widetilde{\mathcal{M}}\left(f_{1}, f_{2}\right), h\right\rangle \gtrsim \delta^{\frac{3(n-1)}{2}} .
$$

The sparse domination of $\left\langle\widetilde{\mathcal{M}}\left(f_{1}, f_{2}\right), h\right\rangle$ yields

$$
\delta^{\frac{3(n-1)}{2}} \leq \delta^{\frac{n+1}{2 r_{1}}} \delta^{\frac{n+1}{2 r_{2}}} \delta^{\frac{n-1}{2 t}} .
$$


This gives us the condition

$$
\frac{n+1}{r_{1}}+\frac{n-1}{s_{1}}+\frac{n+1}{r_{2}}+\frac{n-1}{s_{2}} \leq 4(n-1) .
$$

Therefore, both of $\left(\frac{1}{r_{i}}, \frac{1}{s_{i}}\right), i=1,2$, cannot lie above the line segment $P_{3} P_{4}$ in Figure 1.

Also, the conditions $\frac{1}{r_{i}}+\frac{n}{t} \leq n, i=1,2$, must be satisfied for the sparse domination of the full maximal function as they hold for the lacunary maximal function. Further, by considering $f_{1}=\chi_{R_{1}}, f_{2}=\chi_{B\left(\left(0,0, \ldots, \frac{4}{3}\right), 2\right)}$ and $h=\chi_{R_{2}}$, we obtain that

$$
\delta^{n-1} \lesssim\left\langle\mathcal{M}_{\text {full }}\left(f_{1}, f_{2}\right), h\right\rangle \lesssim \delta^{\frac{n+1}{2 r_{1}}} \delta^{\frac{n-1}{2 t}} .
$$

Therefore, we get that

$$
\frac{n+1}{r_{1}}+\frac{n-1}{t} \leq 2(n-1)
$$

Interchanging the roles of $f_{1}$ and $f_{2}$, we also have that

$$
\frac{n+1}{r_{2}}+\frac{n-1}{t} \leq 2(n-1) \text {. }
$$

These are necessary conditions on various parameters in order the sparse domination to hold for the full bilinear spherical maximal function.

\section{ACKNOWLEDGEMENTS}

The authors are thankful to the referees for their meticulous reading of the manuscript and the very useful remarks, which led to a great improvement of the paper.

All the three authors would like to thank Kangwei Li, José María Martell, and Zoe Nieraeth for their suggestions and comments.

The first author is supported by the Basque Government through the BERC 2018-2021 program and by the Spanish State Research Agency through BCAM Severo Ochoa excellence accreditation SEV-2017-2018 and through project PID2020-113156GB-I00 / AEI / 10.13039/501100011033 and acronym "HAPDE". She also acknowledges the RyC project RYC2018-025477-I and Ikerbasque. The second author acknowledges the financial support from the Science and Engineering Research Board (SERB), Government of India, under the grant MATRICS: MTR/2017/000039/Math. The third author is supported by CSIR (NET), file no. 09/1020 (0094)/2016-EMR-I.

\section{REFERENCES}

[1] T. C. Anderson; E. A. Palsson, Bounds for discrete multilinear spherical maximal functions in higher dimensions, Bull. London Math. Soc. (to appear) and available at arXiv:1910.12458.

[2] S. Bagchi; S. Hait; L. Roncal; S. Thangavelu, On the maximal function associated to the lacunary spherical means on the Heisenberg group, New York J. Math 27 (2021), 631-675.

[3] J. A. Barrionuevo; L. Grafakos; D. He; P. Honzík; L. Oliveira, Bilinear spherical maximal function, Math. Res. Lett. 25 (2018), no. 5, 1369-1388.

[4] J. Bourgain, Averages in the plane over convex curves and maximal operators, J. Anal. Math. 47 (1986), 69-85.

[5] A. P. Calderón; A. Zygmund, A note on the interpolation of linear operations, Studia Math. 12 (1951), 194-204.

[6] C. P. Calderón, Lacunary spherical means, Illinois J. Math. 23 (1979), no. 3, 476-484.

[7] R. R. Coifman; Y. Meyer, Commutateurs d'intégrales singulières et opératerus multilinéaires, Ann. Inst. Fourier (Grenoble) 28 (1978), 177-202.

[8] R. R. Coifman; G. Weiss, Book review: Littlewood-Paley and multiplier theory, Bull. Amer. Math. Soc. 84 (1978), no. 2, 242-250.

[9] M. Cowling; J. G. Cuerva; H. Gunawan, Weighted estimates for fractional maximal functions related to spherical means, Bull. Austral. Math. Soc. 66 (2002), no. 1, 75-90.

[10] W. Damián; A. Lerner; C. Pérez, Sharp weighted bounds for multilinear maximal functions and CalderónZygmund operators, J. Fourier Anal. Appl. 21 (2015), no. 1, 161-181.

[11] G. Dosidis, Multilinear spherical maximal function, Proc. Amer. Math. Soc. 149 (2021), no. 4, 1471-1480.

[12] J. Duoandikoetxea; A. Moyua; O. Oruetxebarria, The spherical maximal operator on radial functions, J. Math. Anal. Appl. 387 (2012), no. 2, 655-666.

[13] J. Duoandikoetxea; L. Vega, Spherical means and weighted inequalities, J. London Math. Soc. (2) 53 (1996), no. $2,343-353$. 
[14] D. A. Geba; A. Greenleaf; A. Iosevich; E. A. Palsson; E. Sawyer, Restricted convolution inequalities, multilinear operators and applications, Math. Res. Lett. 20 (2013), no. 4, 675-694.

[15] L. Grafakos; D. He; P. Honzík, Maximal operators associated with bilinear multipliers of limited decay, J. Anal. Math. (to appear) and available at arXiv:1804.08537.

[16] L. Grafakos; R. H. Torres, Multilinear Calderón-Zygmund theory. Adv. Math. 165 (2002), no. 1, $124-164$.

[17] Y. Heo; S. Hong; C. W. Yang, Improved bounds for the bilinear spherical maximal operators, Math. Res. Lett. 27 (2020), no. 2, 397-434.

[18] E. Jeong; S. Lee, Maximal estimates for the bilinear spherical averages and the bilinear Bochner-Riesz operators, J. Funct. Anal. 279 (2020), no. 7, 108629, 29 pp.

[19] K. Jotsaroop; S. Shrivastava; K. Shuin, Weighted estimates for bilinear Bochner-Riesz means at the critical index, Potential Anal. (to appear).

[20] M. T. Lacey, Sparse bounds for spherical maximal functions, J. Anal. Math. 139 (2019), no. 2, 613-635.

[21] M. T. Lacey; E. Sawyer; I. Uriarte-Tuero, Two weight inequalities for discrete positive operators, arXiv:0911.3437v4.

[22] M. T. Lacey; C. Thiele, $L^{p}$ estimates on the bilinear Hilbert transform for $2<p<\infty$, Ann. of Math.(2) 146 (1997), no. 3, 693-724.

[23] M. T. Lacey; C. Thiele, On Calderón's conjecture, Ann. of Math.(2) 149 (1999), no. 2, 475-496.

[24] A. K. Lerner; F. Nazarov, Intutive dyadic calculus: the basics, Expo. Math. 37 (2019), no. 3, $225-265$.

[25] A. K. Lerner; S. Ombrosi; C. Pérez; R. H. Torres; R. Trujillo-González, New maximal functions and multiple weights for the multilinear Calderón-Zygmund theory, Adv. Math. 220 (2009) 1222-1264.

[26] K. Li; J. M. Martell; H. Martikainen; S. Ombrosi; E. Vuorinen, End-point estimates, extrapolation for multilinear Muckenhoupt classes, and applications, Trans. Amer. Math. Soc. 374 (2021), no.1, 97-135.

[27] K. Li; J. M. Martell; S. Ombrosi, Extrapolation for multilinear Muckenhoupt classes and applications to the bilinear Hilbert transform, Adv. Math. 373 (2020) 17286, 43 pp.

[28] R. Manna, Weighted inequalities for spherical maximal operator, Proc. Japan Acad. Ser. A Math. Sci. 91 (2015), no. 9, 135-140.

[29] G. Mockenhaupt; A. Seeger; C. Sogge, Wave front sets, local smoothing and Bourgain's circular maximal theorem, Ann. of Math. 136 (1992), 207-218.

[30] Z. Nieraeth, Quantitative estimates and extrapolation for multilinear weight classes, Math. Ann. 375 (2019), no. 1-2, 453-507.

[31] X. Shi; Q. Sun, Weighted norm inequalities for Bochner-Riesz operators and singular integral operators. Proc. Amer. Math. Soc. 116 (1992), no. 3, 665-673.

[32] E. M. Stein, Maximal functions. I. Spherical means, Proc. Nat. Acad. Sci. U.S.A. 73 (1976), no. 7, $2174-2175$.

(Saurabh Shrivastava and Kalachand Shuin) Department of Mathematics, Indian Institute Science EducAtion ANd Research Bhopal, Bhopal-462066, India

Email address: $\{$ saurabhk, kalachand16\}@iiserb.ac.in

(Luz Roncal) BCAM - Basque Center for Applied Mathematics, 48009 Bilbao, Spain, Ikerbasque,

Basque Foundation for Science, 48011 Bilbao, Spain

Email address: lroncal@bcamath.org 